\title{
Caching (Bivariate) Gaussians
}

\author{
Giel J. Op ‘t Veld and Michael Gastpar, Fellow, IEEE
}

\begin{abstract}
Caching is a technique that alleviates networks during peak hours by transmitting partial information before a request for any is made. In a lossy setting of Gaussian databases, we study a single-user model in which good caching strategies minimize the data still needed on average once the user requests a file. The encoder decides on a caching strategy by weighing the benefit from two key parameters: the prior preference for a file and the correlation among the files. Considering uniform prior preference but correlated files, caching becomes an application of Wyner's common information and Watanabe's total correlation. We show this case triggers a split: caching Gaussian sources is a non-convex optimization problem unless one spends enough rate to cache all the common information between files. Combining both correlation and user preference we explicitly characterize the full trade-off when the encoder uses Gaussian codebooks in a database of two files: we show that as the size of the cache increases, the encoder should change strategy and increasingly prioritize user preference over correlation. In this specific case we also address the loss in performance incurred if the encoder has no knowledge of the user's preference and show that this loss is bounded.
\end{abstract}

Index Terms-Gaussian Source Coding, Caching, Gray-Wyner network, Common Information, Total Correlation,

\section{INTRODUCTION}

Streaming video services have been a step change in content delivery. The 'on-demand' aspect in particular has been a leap in user experience and a headache for communication engineers. The instantaneous and personalized properties of videoon-demand cause network traffic to become more concentrated in 'internet rush hour', the evening peak when users log in simultaneously. Network and server capacity could suffer from this imbalance; they must be installed to withstand peak traffic, not average. From that perspective, on-demand streaming is both costly and inefficient.

The engineering challenge is to design communication technology that can provide users with the freedom of 'on demand' streaming, while keeping a balanced network load for providers. It would help if servers could anticipate demand and communicate data before it is even requested. In that regard, one cannot help but notice that in this same time frame caching became a popular topic in the information theory community. The most popular caching paper by Maddah-Ali and Niesen

Manuscript received December, 2017; revised September, 2019; accepted May 17, 2020. This work has been supported in part by the Swiss National Science Foundation (FNS grant No. 200021_169294). This paper was presented in part at the 2015 and 2017 WIC Symposium on Information Theory and Signal Processing in the Benelux, as well as the 2016 Annual Conference on Information Science and Systems (CISS), Princeton, USA.

G.J. Op 't Veld was and M.C. Gastpar is with the School of Computer and Communication Sciences, École Polytechnique Fédéerale de Lausanne, 1015 Lausanne, Switzerland (e-mail: giel.optveld@alumni.epfl.ch, michael.gastpar@epfl.ch).

Copyright (c) 2017 IEEE. Personal use of this material is permitted. However, permission to use this material for any other purposes must be obtained from the IEEE by sending a request to pubs-permissions@ieee.org
[1] was first published on arXiv in the same year in which Netflix tripled her stock value [2].

This paper studies the coding of information in two phases in a one-user bubble: first to anticipate demand by writing something in the cache of the user, followed by responding to an actual request for data. While the second phase is often called delivery, we prefer the term update. Most likely, some data stored in the cache will turn out to be redundant once a user transmits an actual request. This loss is accepted in the knowledge that otherwise waiting for a user to make a request would burden the network during the 'peak hours'. The question is whether despite this loss there are still smart strategies to reduce overall communication rates.

We study these problems in a lossy source coding setting and, even more specific, modeling databases by (possibly correlated) Gaussian sources [3]-[5]. The model (Figure 2a) is based on the (single user) work of Wang et al [6] who ventured in this direction using discrete information sources. Recently this model was extended to a lossy setting as well by Timo et al [7]. Their focus was on designing caching strategies that optimize the worst-case update scenario. Our objective, however, will be to design caching codes that do well on average and to -when possible- also model asymmetric user preference for certain data. In our model, the cache encoder weighs two parameters in its strategy: the preference a user has for some files in the database versus the correlation between those files (perhaps the encoder can transmit information that is common to the files so the cache is useful no matter the user's request).

The Wang-Lim-Gastpar caching model is closely related to the classic Gray-Wyner network (Figure 2b), in which an encoder communicates two files to two decoders (one for each) via one common and two individual communication links [8]. The first stands analogous to the cache that gets transmitted in any case, whereas the individual ones can be viewed upon as the update messages that will be sent if either one file or the other is requested. We will argue that caching without a bias in user preference is closely related to the notions of Wyner's common information [9] and Watanabe's total correlation [10]. Research that is closely related to this work therefore also includes the work of Viswanatha et al [11] and $\mathrm{Xu}$ et al [12]. These authors have introduced and studied notions of lossy common information, as well as provided some important characterizations of these properties for Gaussian distributions.

\section{A. Summary of Results}

We present a lossy caching model (Figure 2a) based on the Gray-Wyner network [8], similar to [6], [7]. Our model distinguishes itself by focusing on average performance and also including user preference. We provide a full characterization 
of achievable caching strategies based on the analogy to the Gray-Wyner network in Theorem 1 for bivariate and Theorem 5 for multivariate databases.

Our main aim is to delve one level deeper: what should caching strategies actually look like when we take our information sources to be Gaussians? Restricting to using also Gaussian codebooks, we characterize optimal strategies through what we call the Gaussian caching rate-distortion function (Corollaries 1 and 8).

The structure of the paper is to first study a database of two Gaussian sources as a self-contained story and to afterwards discuss what changes for larger databases. This choice is due to the Gaussian rate-distortion function subject to individual mean squared errors: that function is known in analytic form for bivariates, but only as an (numerically solvable) optimization problem for multivariates. In the former case, it is hence possible to attain a greater level of detail and closed-form insight.

Overall we determine two key drivers: user preference and the correlation among the information sources. Our main contributions can be summarized as follows:

- Bivariates / Databases of two Gaussian sources:

- When the user's choice is uniform, but the sources are dependent: we show in Section $\mathrm{V}$ that the encoder should perform caching via a reverse waterfilling procedure on the correlation matrix (not the covariance).

- When the user's choice is non-uniform and the sources are dependent we derive in Section VI the optimal caching strategy over all Gaussian codebooks. In addition, we discuss in Section VII how finding caching strategies depends on the size of the cache, how it is not a successively refinable process and lastly we bound rate loss due to absence of knowledge on user preference.

- Multivariates / Databases of multiple Gaussian sources:

- Section VIII extends the bivariate model to larger databases and characterizes the achievable rate region through Theorem 5 and specifically for Gaussian sources and codebooks in Corollary 8.

- When the user's choice is uniform, but the sources are dependent: Section VIII-C proves that when the cache is large then identifying and caching all common information between the files is a convex problem (Theorems 6 and 7). When the cache is small, emulating the optimal strategy for bivariates only serves as an inner bound for larger databases (Lemma 8).

\section{B. Related Literature}

There is a substantial amount of information-theoretic literature on coded caching at this point. The focus is usually on scenarios with multiple users, by contrast to our work. The issue of caching for non-uniform file preferences has been considered starting with [13], where bounds are derived for a multi-user scenario in a worst-case sense with lossless file reconstruction, and in [14], where an order-optimal scheme is
TABLE I: Most used matrix and vector functions.

$\begin{array}{ll}\text { Notation } & \text { Meaning } \\ \lambda_{i}(\mathbf{A}) & \text { the } i \text { 'th eigenvalue of } \mathbf{A} . \\ \lambda_{\min }, \lambda_{\max } & \text { the smallest and respectively largest eigenvalue. } \\ \operatorname{diag}(\mathbf{A}) & \text { a vector containing the diagonal elements of the matrix } \mathbf{A} . \\ \operatorname{diag}(\mathbf{v}) & \text { a diagonal matrix of non-zero entries equal to the vector } \mathbf{v} . \\ |\mathbf{A}| & \text { the determinant of } \mathbf{A} . \\ \|\mathbf{v}\| & \text { the Euclidean norm of the vector } \mathbf{v} . \\ \operatorname{ker}(\mathbf{A}) & \text { the kernel (or nullspace) of } \mathbf{A} . \\ \operatorname{dim}(\cdot) & \text { the dimension of a vector space. }\end{array}$

given under the same model. The issue of caching for correlated files has been considered in the lossless case in [15], [16], in the setting of lossless file reconstruction. Furthermore, some studies simultaneous to ours have also considered Gaussian models of files. For example, [17] presents an experimental simulation-driven study of Gaussian caching in a broadcast scenario to multiple users. Moreover, [18] considers independent Gaussian file models and derives rate results under a worst-case (request) model with multiple users, letting the distortion constraints vary per user.

\section{Preliminaries}

\section{A. Notation}

The $i$-th file of our database is a length- $N$ sequence of i.i.d. samples $X_{i}^{N}$. The database is a collection of $K=2$ of these Gaussian sequences, denoted in vector notation like $\mathbf{X}^{N}$. Although the samples are drawn i.i.d. in time, $X_{i}^{n}$ and $X_{j}^{n}$ can be dependent. One can therefore equivalently say that the database $\mathbf{X}^{N}$ is a sequence of i.i.d. vector samples drawn via a Gaussian multivariate $\mathbf{X}$ distribution $\sim \mathcal{N}\left(\mathbf{0}, \Sigma_{\mathbf{X}}\right)$. Unless explicitly stated otherwise, this covariance matrix $\Sigma_{\mathbf{X}}$ decompose as follows,

$$
\Sigma_{\mathbf{X}}=\left[\begin{array}{cc}
\sigma_{1}^{2} & \rho_{12} \sigma_{1} \sigma_{2} \\
\rho_{12} \sigma_{1} \sigma_{2} & \sigma_{2}^{2}
\end{array}\right]
$$

where $\sigma_{i}$ is the variance of $X_{i}$, and $\rho_{i j}$ the correlation between $X_{i}$ and $X_{j}$. More so than not will unit variance be assumed for simplicity.

Boldface letters are reserved for either vectors (like $\mathbf{v}$ ) or matrices (like A). Capital letters denote either matrices, random variables or random vectors (like $\mathbf{A}, X$ and $\mathbf{X}$ respectively). To avoid confusion between matrices and random vectors, the end of the alphabet is reserved for random variables. $A_{i, j}$ is a single element of the matrix A. As working with Gaussians requires a fair amount of linear algebra, Table I summarizes the most used operators. The semidefinite ordering symbol $\mathbf{A} \preceq \mathbf{B}$ means $\mathbf{B}-\mathbf{A}$ is positive semidefinite. The strict inequality $\prec$ implies the difference of both matrices is positive definite.

Logarithms are all base-2, and $\log ^{+}(x)=\max (0, \log (x))$; the superscript ${ }^{+}$is also used on other occasions to enforce non-negativity. The set $[a b]$ with square brackets indicates the set of all elements between $a$ and $b$, whereas $\{a, b\}$ contains only $a$ and $b$. Differential entropy is denoted by $h(X)$ and $I(X ; Y)$ stands for mutual information. Lastly, $X-Y-Z$ signifies a Markov chain. 


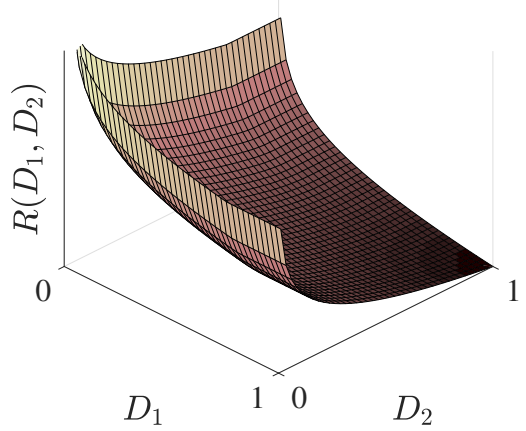

(a) $R\left(D_{1}, D_{2}\right)$.

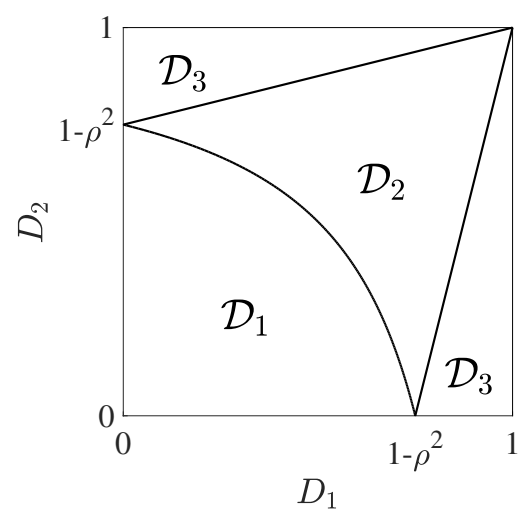

(b) The distortion plane as defined by (6)-(8).

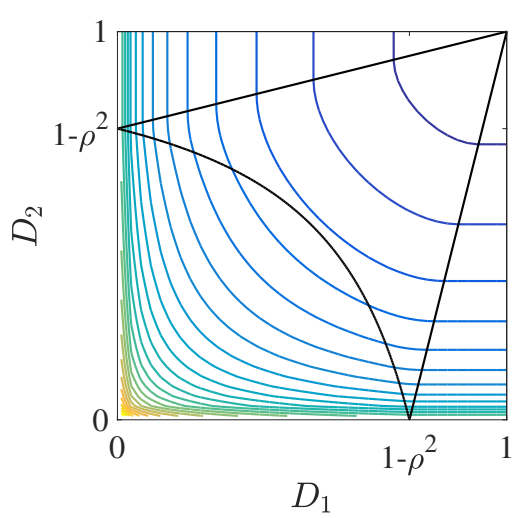

(c) The contour lines of $R\left(D_{1}, D_{2}\right)$.

Fig. 1: Visualization of the bivariate Gaussian joint rate-distortion function and its different operating regions.

\section{B. The Gaussian (Bivariate) Rate-Distortion Function}

A crucial tool in this work is the Gaussian rate-distortion function with respect to individual squared error. This section is intended to set notation and in particular to recap the closed form expression for bivariate Gaussians for reference in later sections. Let $\mathbf{d}$ be the vector of $K$ individual mean-squared error criteria $D_{1}, D_{2}, \cdots, D_{K}$. Then define the joint ratedistortion function as follows:

$$
\begin{aligned}
R(\mathbf{d})=\min & I(\mathbf{X}, \hat{\mathbf{X}}) \\
\text { s.t. } & \mathbb{E}\left[\left(X_{i}-\hat{X}_{i}\right)^{2}\right] \leq D_{i}, \quad i=1, \cdots, K
\end{aligned}
$$

Xiao and Luo derived a simpler form for this function for Gaussian distributed $\mathbf{X}$, expressing it as a semidefinite programming problem [19]. First, let $\mathbf{D}$ denote the mean squared error matrix between $\mathbf{X}$ and the lossy representation $\hat{\mathbf{X}}$ :

$$
\mathbf{D} \triangleq \mathbb{E}\left[(\mathbf{X}-\hat{\mathbf{X}})(\mathbf{X}-\hat{\mathbf{X}})^{T}\right]
$$

Then, the Xiao-Luo form of expressing the Gaussian ratedistortion function is the following:

$$
R(\mathbf{d})=\min _{\mathbf{D}} \frac{1}{2} \log \frac{\left|\Sigma_{\mathbf{X}}\right|}{|\mathbf{D}|} \quad \text { s.t. }\left\{\begin{array}{l}
\mathbf{0} \preceq \mathbf{D} \preceq \Sigma_{\mathbf{X}} \\
\operatorname{diag}(\mathbf{D}) \leq \mathbf{d} .
\end{array}\right.
$$

Let $K=2$ and assume w.1.o.g. that $\sigma_{1}^{2}=\sigma_{2}^{2}=1$. Then for distortions $0 \leq D_{1}, D_{2} \leq 1$, the bivariate rate-distortion function reduces to:

$$
\begin{aligned}
& R\left(D_{1}, D_{2}\right)= \\
& \begin{cases}\frac{1}{2} \log \left(\frac{1-\rho^{2}}{D_{1} D_{2}}\right) & \text { if }\left(D_{1}, D_{2}\right) \in \mathcal{D}_{1}, \\
\frac{1}{2} \log \left(\frac{1-\rho^{2}}{D_{1} D_{2}-\left(|\rho|-\sqrt{\left(1-D_{1}\right)\left(1-D_{2}\right)}\right)^{2}}\right) & \text { if }\left(D_{1}, D_{2}\right) \in \mathcal{D}_{2} \\
\frac{1}{2} \log \left(\frac{1}{\min \left(D_{1}, D_{2}\right)}\right) & \text { if }\left(D_{1}, D_{2}\right) \in \mathcal{D}_{3}\end{cases}
\end{aligned}
$$

where

$$
\begin{aligned}
\mathcal{D}_{1}= & \left\{D_{1}, D_{2}:\left(1-D_{1}\right)\left(1-D_{2}\right) \geq \rho^{2}\right\} \\
\mathcal{D}_{2}= & \left\{D_{1}, D_{2}:\right. \\
& \left.\left(1-D_{1}\right)\left(1-D_{2}\right) \leq \rho^{2} \leq \min \left(\frac{1-D_{1}}{1-D_{2}}, \frac{1-D_{2}}{1-D_{1}}\right)\right\}, \\
\mathcal{D}_{3}= & \mathcal{D}_{1}^{c} \cap \mathcal{D}_{2}^{c} .
\end{aligned}
$$

We refer to this segmentation of different $\left(D_{1}, D_{2}\right)$ as the $\mathcal{D}$-plane or the distortion plane. Figure 1 attempts to make the above expressions tangible through visualization. If the sources are not of unit variance then one has to normalize each $X_{i}$ and scale the respective $D_{i}$ accordingly.

Lastly, we remind the reader that the single Gaussian ratedistortion function subject to a squared error is

$$
R(D)=\frac{1}{2} \log \frac{\sigma^{2}}{D} \text {. }
$$

General notation is $R(D)$. Adding a subscript like $R_{X_{i}}(D)$ is reserved for drawing explicit attention to which distribution constitutes the rate-distortion function if required so by context.

\section{Problem Statement}

Figure $2 \mathrm{a}$ serves as the model to capture the essence of caching. The database consists of two 'files', $X_{1}^{N}$ and $X_{2}^{N}$, and there are two encoders: One encoder (the "cache") is unaware of which of the two files is requested, while the other encoder knows which file is requested (the "update"). We suppose that with probability $p$, it is file $X_{1}^{N}$ that is being requested, and thus with probability $1-p$, it is file $X_{2}^{N}$. Our goal is to understand the trade-offs between the code rate of the "cache" encoder and the (average) code rate of the "update" encoder.

\section{A. The Caching Network}

Let us now formally state our problem. Let $\mathbf{X}^{N}$ be a sequence of two-dimensional random vectors which fulfills the role of the database in our model. Each sample of $\mathbf{X}$ is drawn in an i.i.d. fashion, but within one sample the vector elements $X_{1}$ and $X_{2}$ can be correlated. This sequence is to be encoded into three messages, $m_{c}$ for the 'cache' and $m_{u, 1}, m_{u, 2}$ for the 'update'. The cache message $m_{c}$ is transmitted in any case, while for the update the decoder will only receive $m_{u, 1}$ if it requests $X_{1}^{N}$ (and similar for $m_{u, 2}$ and $X_{2}^{N}$ ). Each message $m$ is an integer in the set $I_{M}=\{1,2, \cdots, M\}$, where the set size for each message is denoted by $M_{c}, M_{u, 1}$ and $M_{u, 2}$.

A code consists of encoder mappings

$$
f: \quad \mathbb{R}^{2 \times N} \rightarrow I_{M_{c}} \times I_{M_{u, 1}} \times I_{M_{u, 2}}
$$




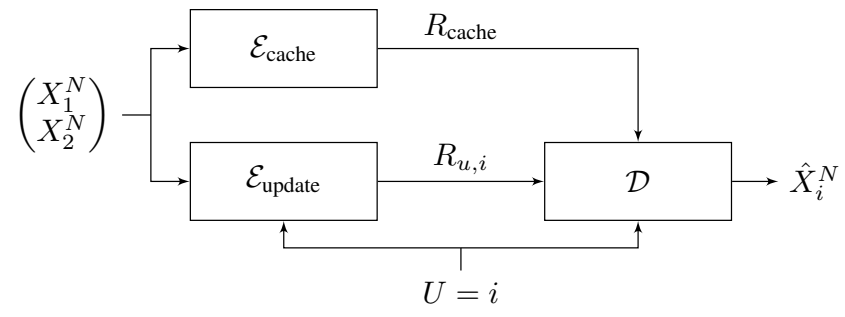

(a) The caching network, similar to [6].

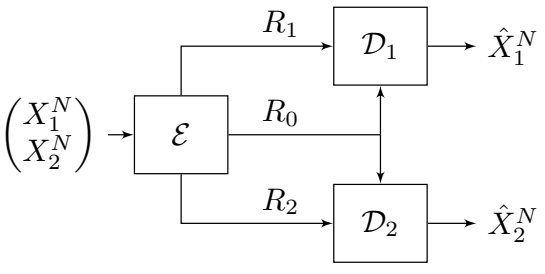

(b) The classic Gray-Wyner network [8].

Fig. 2: The caching network resembles the Gray-Wyner network by drawing each update scenario as a separate link.

and a decoder

$$
g_{i}: \quad I_{M_{c}} \times I_{M_{u, i}} \rightarrow \mathbb{R}^{1 \times N} \quad \text { for } i=1,2
$$

of which the latter is meant to reconstruct $\hat{X}_{i}^{N}=g_{i}\left(m_{c}, m_{u, i}\right)$ where $i$ stands for the specific file requested.

A caching rate-distortion tuple $\left(R_{\text {cache }}, R_{u, 1}, R_{u, 2}, D_{F}\right)$ is said to be achievable if for arbitrary $\epsilon>0$ there exist such encoders and decoders that for both $i=1,2$ we have

$$
\begin{aligned}
M_{c} & \leq 2^{N\left(R_{\text {cache }}+\epsilon\right)} & & \\
M_{u, i} & \leq 2^{N\left(R_{u, i}+\epsilon\right)} & & \text { for } i=1,2 \\
\frac{1}{N} \sum_{n=1}^{N} d\left(X_{i}(n), \hat{X}_{i}(n)\right) & \leq D_{F}+\epsilon & & \text { for } i=1,2
\end{aligned}
$$

where $d_{X}(\cdot, \cdot)$ is some single-letter distortion measure. As is clear from the definition, we apply symmetric end distortion criteria to $X_{1}$ and $X_{2}$. This is a matter of notational convenience and it will later become clear that (a)symmetry is neither important nor interesting. More relevant to study in the Gaussian case are the intermediate distortion levels after caching, these will be introduced in Section III-D.

After the caching phase, the user submits a requests for either $X_{1}^{N}$ or $X_{2}^{N}$ which is modeled by the Bernoulli random variable $U \in\{1,2\}$, distributed as $P(U=1)=p$. The main question we pose is: What does one need to cache in order to minimize the update rate that is still needed on average? To that end define also the average update rate:

$$
\bar{R}_{\text {update }} \triangleq p R_{u, 1}+(1-p) R_{u, 2} .
$$

A shorthand notation will be to say that $\left(R_{\text {cache }}, \bar{R}_{\text {update }}, D_{F}\right)$ is achievable to indicate that (at least one tuple) ( $R_{\text {cache }}, R_{u, 1}, R_{u, 2}, D_{F}$ ) is, of which the average update rate equals $\bar{R}_{\text {update }}$.

\section{B. Analogy to the Gray-Wyner Network}

From an operational perspective and the existence of codes, there is a complete equivalence to the Gray-Wyner network [8], as depicted in Figure 2b. Namely, even though the decoder is only interested in $X_{1}^{N}$ or $X_{2}^{N}$, any code should be capable of doing both as the user could request any of the two. By taking Figure $2 \mathrm{a}$ and drawing the events of the user asking for $X_{1}$ or $X_{2}$ as two separate decoders one obtains $2 \mathrm{~b}$. The equivalence is thus as follows:

$$
R_{0} \leftrightarrow R_{\text {cache }}, \quad R_{1} \leftrightarrow R_{u, 1}, \quad R_{2} \leftrightarrow R_{u, 2} .
$$

The Gray-Wyner network was introduced originally in [8], but also featured more recently in an explicit lossy source coding setting [11], [12]. The region of achievable ratedistortion tuples on the Gray-Wyner network is the union of all $\left(R_{0}, R_{1}, R_{2}, D_{1}, D_{2}\right)$ satisfying

$$
\begin{aligned}
& R_{0} \geq I(\mathbf{X} ; V) \\
& R_{1} \geq I\left(X_{1} ; \hat{X}_{1} \mid V\right) \\
& R_{2} \geq I\left(X_{2} ; \hat{X}_{2} \mid V\right) \\
& D_{1} \geq \mathbb{E}\left[d_{X_{1}}\left(X_{1}, \hat{X}_{1}\right)\right] \\
& D_{2} \geq \mathbb{E}\left[d_{X_{2}}\left(X_{2}, \hat{X}_{2}\right)\right]
\end{aligned}
$$

over joint densities $p(\mathbf{x}, v, \hat{\mathbf{x}})$, for some distortion measures $d_{X}(\cdot, \cdot)$. Notation-wise, $V$ may be a single random variable, or also a vector, but for consistency (and with the end result in mind) we stick to denoting $V$ by a non-bold character.

Hence, one knows which caching strategies are achievable:

Theorem 1. A caching rate-distortion tuple $\left(R_{\text {cache }}, \bar{R}_{\text {update }}, D_{F}\right)$ is achievable if and only if there exists $a V$ and a joint probability density $p(\mathbf{x}, v, \hat{\mathbf{x}})$ such that the following inequalities are satisfied:

$$
\begin{aligned}
R_{\text {cache }} & \geq I(\mathbf{X} ; V) \\
\bar{R}_{\text {update }} & \geq p I\left(X_{1} ; \hat{X}_{1} \mid V\right)+(1-p) I\left(X_{2} ; \hat{X}_{2} \mid V\right) \\
D_{F} & \geq \mathbb{E}\left[d_{X_{i}}\left(X_{i}, \hat{X}_{i}\right)\right], \text { for } i=1,2
\end{aligned}
$$

The closure of such achievable tuples over joint densities $p(\mathbf{x}, v, \hat{\mathbf{x}})$ is denoted $\mathcal{R}_{\text {caching. }}$.

Proof. The achievability region follows directly from the equivalence with the Gray-Wyner network (11) and taking the average over the individual links (10).

The goal is now to better understand the boundary of $\mathcal{R}_{\text {caching, }}$, to understand which strategies are not only achievable, but are also good.

\section{Characteristics and (Non-)Attainable Limits}

For a fixed $D_{F}$, the boundary of the caching rate-distortion region of Theorem 1 is a curve that is convex in $R_{\text {cache }}$ and lies inside the triangle depicted in Figure 3. That shape is the intersection of the following three bounds. Recall for these equations that $R(D)$ is the single rate-distortion function and $R\left(D_{1}, D_{2}\right)$ is the bivariate rate-distortion function subject to individual distortion constraints. Figure 3 is then built as follows: 


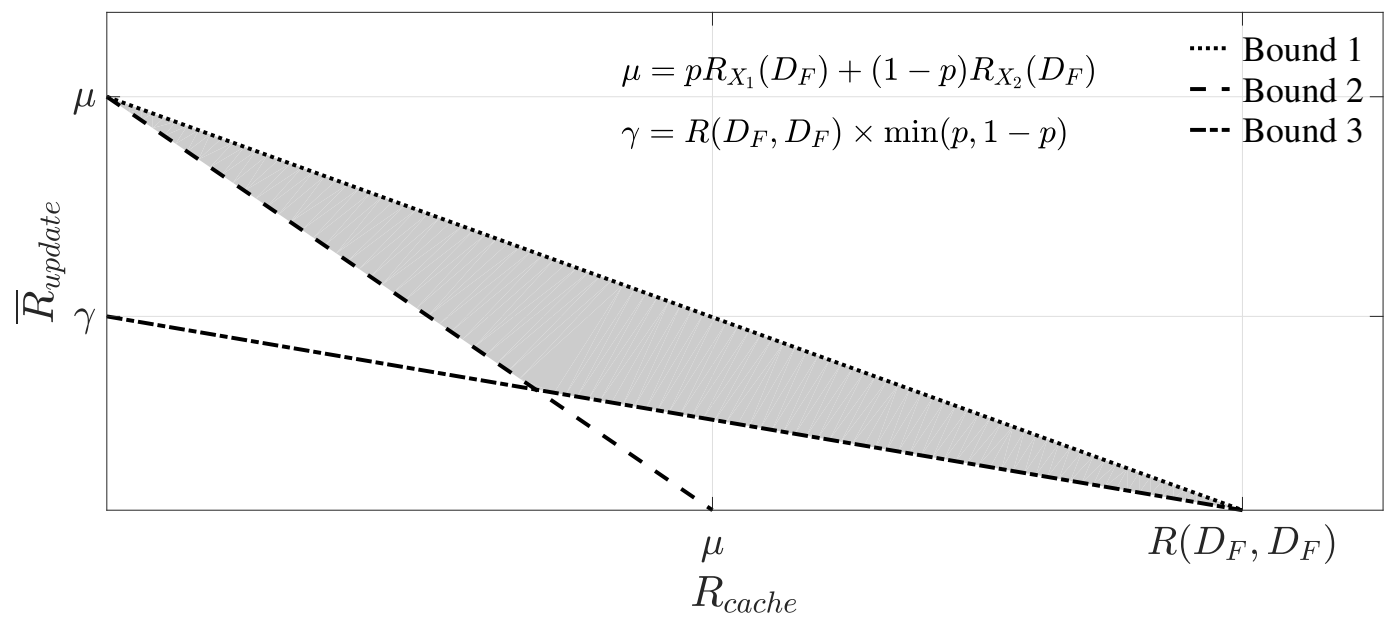

Fig. 3: The boundary of achievable $\left(R_{\text {cache }}, \bar{R}_{\text {update }}\right)$-pairs lies inside this gray triangle, whose bounds are labeled according to their order in Section III-C.

1) Bound 1 in Figure 3 is an achievable inner bound based on time-sharing the extremal strategies of caching both files completely in advance or sending only one file completely in the update phase by waiting for the user to make a request:

update everything:

$$
\left(R_{\text {cache }}, \bar{R}_{\text {update }}\right)=\left(0, p R_{X_{1}}\left(D_{F}\right)+(1-p) R_{X_{2}}\left(D_{F}\right)\right)
$$

or cache everything:

$$
\left(R_{\text {cache }}, \bar{R}_{\text {update }}\right)=\left(R\left(D_{F}, D_{F}\right), 0\right) \text {. }
$$

Here, $R_{X_{i}}(D)$ denotes the standard rate-distortion function of $X_{i}$ with respect to mean-squared error, evaluated at $D$, see Equation (9) and the ensuing discussion.

2) Bound 2 in Figure 3 is an outer bound connecting the two points:

$$
\begin{aligned}
& \left(R_{\text {cache }}, \bar{R}_{\text {update }}\right)=\left(0, p R_{X_{1}}\left(D_{F}\right)+(1-p) R_{X_{2}}\left(D_{F}\right)\right) \\
& \left(R_{\text {cache }}, \bar{R}_{\text {update }}\right)=\left(p R_{X_{1}}\left(D_{F}\right)+(1-p) R_{X_{2}}\left(D_{F}\right), 0\right),
\end{aligned}
$$

of which the first point is achievable with certainty. The bound stems from the following inequality:

$$
\begin{aligned}
R_{\text {cache }}+\bar{R}_{\text {update }}= & p\left(R_{\text {cache }}+R_{u, 1}\right) \\
& +(1-p)\left(R_{\text {cache }}+R_{u, 2}\right) \\
\geq & p R_{X_{1}}\left(D_{F}\right)+(1-p) R_{X_{2}}\left(D_{F}\right) .
\end{aligned}
$$

If $R_{\text {cache }}=0$, then all communication happens in the update phase when the encoder is aware of the user's request. Therefore indeed the leftmost point of this outer bound coincides with the start of the boundary of achievable $\left(R_{\text {cache }}, \bar{R}_{\text {update }}\right)$ - pairs.

3) Bound 3 in Figure 3 is an outer bound connecting the two points:

$$
\begin{aligned}
& \left(R_{\text {cache }}, \bar{R}_{\text {update }}\right)=\left(0, R\left(D_{F}, D_{F}\right)\right) \\
& \left(R_{\text {cache }}, \bar{R}_{\text {update }}\right)=\left(\min (p, 1-p) \times R\left(D_{F}, D_{F}\right), 0\right),
\end{aligned}
$$

of which the second is achievable with certainty. This bound stems from $R_{\text {cache }}+R_{u, 1}+R_{u, 2} \geq R\left(D_{F}, D_{F}\right)$. Equality implies that the joint rate-distortion function can be completely distributed over all branches of the GrayWyner network. This is known to be possible in some cases ( $R_{\text {cache }}$ needs to be large) and definitely not in others (see, e.g., [11]). The caching of Gaussian sources will also have equality for some $\left(R_{\text {cache }}, \bar{R}_{\text {update }}\right)$, as will be detailed later.

\section{The Gaussian Case}

Let us zoom in further: the database $\mathbf{X}^{N}$ are i.i.d. samples from a Gaussian distribution $\sim \mathcal{N}\left(\mathbf{0}, \Sigma_{\mathbf{X}}\right)$ with covariance

$$
\Sigma_{\mathbf{X}}=\left[\begin{array}{ll}
1 & \rho \\
\rho & 1
\end{array}\right] .
$$

Reflecting on the equations of Theorem 1, one must note that the source $\mathbf{X}$ being Gaussian distributed does not imply that $V$ and $\hat{\mathbf{X}}$ are necessarily also Gaussian on the boundary of this region. In Corollaries 2 and 5 we will show that using Gaussian codebooks and sufficiently large $R_{\text {cache }}$ it is possible to attain communication at rates for which it holds that

$$
R_{\text {cache }}+R_{u, 1}+R_{u, 2}=R\left(D_{F}, D_{F}\right) \text {. }
$$

Since one cannot do better than the joint rate-distortion function, Gaussian auxiliaries are thus sufficient for optimality in these cases. However, in general it holds that

$$
R_{\text {cache }}+R_{u, 1}+R_{u, 2} \geq R\left(D_{F}, D_{F}\right)
$$

and it is clear a priori that this condition cannot be met with equality everywhere. For starters, we have in Figure 3 that the outer bound associated to this inequality crosses another outer bound. Whenever the inequality is strict, to the best of our knowledge it is not known whether Gaussian auxiliaries are sufficient for optimality; this remains an open problem. In this work, we restrict ourselves to all variables being jointly Gaussian. From here onwards we therefore speak of the Gaussian achievable caching rate-distortion region. 
Corollary 1. The Gaussian boundary of $R_{\text {caching }}$ can be characterized by

$$
\begin{array}{cl}
R_{\text {cache }}\left(d, D_{F}\right)=\min _{D_{F} \leq D_{1}, D_{2} \leq 1} & R\left(D_{1}, D_{2}\right) \\
\text { s.t. } & D_{1}^{p} D_{2}^{1-p} \leq d
\end{array}
$$

for a normalized parameter $d \in\left[D_{F}, 1\right]$ that relates back to $\bar{R}_{\text {update }}$ by picking $d=D_{F} 2^{2 \bar{R}_{\text {update }}}$.

Proof. A shorthand but equally correct characterization of $\mathcal{R}_{\text {caching }}$ is by means of the conditional rate-distortion function, i.e., to take the union over all $p(\mathbf{x}, v)$ of

$$
\left\{\begin{array}{l}
R_{\text {cache }} \quad \geq I(\mathbf{X} ; V) \\
\bar{R}_{\text {update }} \geq p R_{X_{1} \mid V}\left(D_{F}\right)+(1-p) R_{X_{2} \mid V}\left(D_{F}\right) .
\end{array}\right.
$$

By taking $p(\mathbf{x} \mid v)$ to be Gaussian, also $R_{X_{i} \mid V}\left(D_{F}\right)$ is solved by Gaussian distributions since it is the scalar rate-distortion function (9). Then, the cache rate condition translates to:

$$
\begin{aligned}
R_{\text {cache }} & \geq I(\mathbf{X} ; V) \\
& =\frac{1}{2} \log \frac{\left|\Sigma_{\mathbf{X}}\right|}{|\mathbf{D}|},
\end{aligned}
$$

and the update

$$
\begin{aligned}
\bar{R}_{\text {update }} & \geq p I\left(X_{1} ; \hat{X}_{1} \mid V\right)+(1-p) I\left(X_{2} ; \hat{X}_{2} \mid V\right) \\
& =\frac{p}{2} \log ^{+} \frac{D_{1,1}}{D_{F}}+\frac{1-p}{2} \log ^{+} \frac{D_{2,2}}{D_{F}} \\
& =\frac{1}{2} \log ^{+} \frac{D_{1,1}^{p} D_{2,2}^{1-p}}{D_{F}},
\end{aligned}
$$

where

$$
\mathbf{D}=\mathbb{E}\left[(\mathbf{X}-\mathbb{E}[\mathbf{X} \mid V])(\mathbf{X}-\mathbb{E}[\mathbf{X} \mid V])^{T}\right]=\Sigma_{\mathbf{X} \mid V} .
$$

Any positive semidefinite matrix $\mathbf{D}$ that satisfies $\mathbf{D} \preceq \Sigma_{\mathbf{X}}$ can be associated to a random variable $V$ that is jointly Gaussian with $\mathbf{X}$, and vice versa (see, e.g., [19]). Such a matrix can be interpreted as a mean-squared error distortion after caching, but before the update phase. One can thus equivalently optimize over all $\mathbf{D}$ rather than over all Gaussian distributions $p(\mathbf{x}, v)$.

Now fix one rate and minimize the other:

$$
R_{\text {cache }}(\gamma)=\min R_{\text {cache }} \quad \text { s.t. } \quad \bar{R}_{\text {update }} \leq \gamma .
$$

As a matter of definition, instead of bounding $\bar{R}_{\text {update }}$ one can equivalently bound $D_{1,1}^{p} D_{2,2}^{1-p}$ to emphasize that the distortions up to which one caches the sources are the intrinsic variables of this problem; they are both objective and constraint. Observe also that it serves no purpose to cache either $X_{1}$ or $X_{2}$ beyond the final distortion constraint $D_{F}$ (22). In other words, it is futile to pick a caching distortion profile $\mathbf{D}$ of which $D_{1,1}<D_{F}$ or $D_{2,2}<D_{F}$; that rate is better spent on caching a component that does not yet satisfy the end criterion.

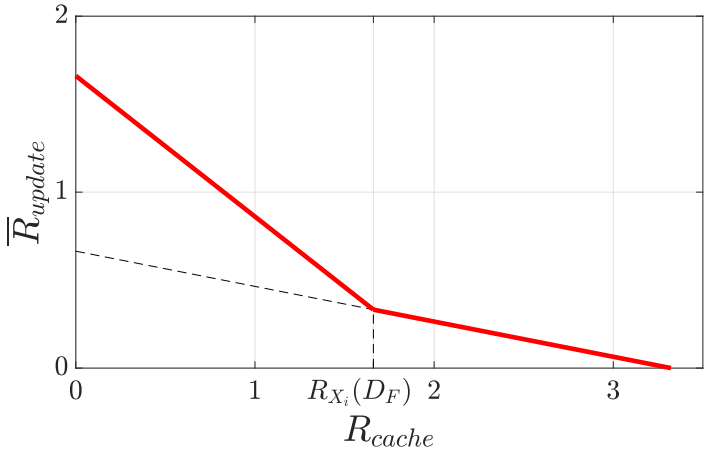

Fig. 4: If $X_{1}$ and $X_{2}$ are independent, the $\left(R_{\text {cache }}, \bar{R}_{\text {update }}\right)$ trade-off is piece-wise linear with slopes $\max (p, 1-p)$ (left) and $\min (p, 1-p)$ (right). The turning point is at $R_{\text {cache }}=$ $R_{X_{i}}\left(D_{F}\right)$, where $X_{i}$ is the most popular file. Drawn with $D_{F}=0.1$.

Combining all, define and simplify the following caching rate-distortion function:

$$
\begin{aligned}
& R_{\text {cache }}\left(d, D_{F}\right)= \min _{\mathbf{D}} \quad \frac{1}{2} \log \frac{\left|\Sigma_{\mathbf{X}}\right|}{|\mathbf{D}|} \\
& \text { s.t. } \quad\left\{\begin{array}{l}
0 \preceq \mathbf{D} \preceq \Sigma_{\mathbf{X}} \\
D_{1,1}^{p} D_{2,2}^{1-p} \leq d \\
D_{1,1}, D_{2,2} \geq D_{F}
\end{array}\right. \\
&=\min _{D_{1}, D_{2} \geq D_{F}} \quad \min _{\mathbf{D}}^{\operatorname{diag}(\mathbf{D})=\left(D_{1}, D_{2}\right)} \\
& \frac{1}{2} \log \frac{\left|\Sigma_{\mathbf{X}}\right|}{|\mathbf{D}|} \\
&=\min _{D_{F} \leq D_{1}, D_{2} \leq 1} \quad \begin{array}{c}
R \preceq \mathbf{D} \preceq \Sigma_{\mathbf{X}} \\
D_{1}^{p} D_{2}^{1-p} \leq d \\
\text { s.t. } \left.\quad D_{1}, D_{2}\right)
\end{array}
\end{aligned}
$$

Note that the caching rate-distortion function is a minimization of a convex function over a non-convex domain. Furthermore, also note that even though we constrain $D_{1}^{p} D_{2}^{1-p} \leq d$, the minimizer to (18) will result in equality by construction.

Definition 1. A caching strategy refers to a pair of intermediate distortions $\left(D_{1}, D_{2}\right)$ after the caching phase. Such a strategy is said to be optimal if it is the minimizer to (18) w.r.t. an instance of $d$ and $D_{F}$.

The extreme ends of (18) like depicted in Figure 3 simplify thanks to the symmetry assumptions (assuming $p \notin\{0,1\}$ ):

$$
\begin{aligned}
R_{\text {cache }}\left(D_{F}, D_{F}\right) & \rightarrow\left(R_{\text {cache }}, \bar{R}_{\text {update }}\right)=\left(R\left(D_{F}, D_{F}\right), 0\right) \\
R_{\text {cache }}\left(1, D_{F}\right) & \rightarrow\left(R_{\text {cache }}, \bar{R}_{\text {update }}\right)=\left(0, R\left(D_{F}\right)\right)
\end{aligned}
$$

In the following sections we aim to understand $R_{\text {cache }}\left(d, D_{F}\right)$ while playing with both the correlation $\rho$ and the file preference $p$. 


\section{PREFEREnCE, BUt NO DEPENDENCE}

We begin our study with the simple case of independent files. It is immediately clear that in this case, the server should cache the more preferred file first (to the desired level of fidelity), before beginning to cache the less preferred file. Formally, we record the following lemma:

Lemma 1. Assume without loss of generality that $p>1-p$, if $X_{1}$ and $X_{2}$ are independent then the boundary of $\mathcal{R}_{\text {caching }}$ is the following connection of two straight lines:

$$
\begin{aligned}
& R_{\text {cache }}=\delta \\
& \bar{R}_{\text {update }}=p\left(R_{X_{1}}\left(D_{F}\right)-\delta_{1}\right)^{+}+(1-p)\left(R_{X_{2}}\left(D_{F}\right)-\delta_{2}\right)^{+} \\
& \text {for } \delta \in\left[0, R_{X_{1}}\left(D_{F}\right)+R_{X_{2}}\left(D_{F}\right)\right] \text { and } \\
& \quad \text { if } \delta \leq R_{X_{1}}\left(D_{F}\right) \text {, then } \\
& \quad\left(\delta_{1}, \delta_{2}\right)=(\delta, 0) \\
& \quad \text { if } R_{X_{1}}\left(D_{F}\right) \leq \delta \leq R_{X_{1}}\left(D_{F}\right)+R_{X_{2}}\left(D_{F}\right) \text {, then } \\
& \quad\left(\delta_{1}, \delta_{2}\right)=\left(R_{X_{1}}\left(D_{F}\right), \delta-R_{X_{1}}\left(D_{F}\right)\right) \\
& \text { If } p<1-p, X_{1} \text { and } X_{2} \text { switch roles. }
\end{aligned}
$$

Proof. Recall this bound from the Gray-Wyner network:

$$
\begin{aligned}
R_{\text {cache }}+R_{u, 1}+R_{u, 2} & \geq R\left(D_{F}, D_{F}\right) \\
& \geq R_{X_{1}}\left(D_{F}\right)+R_{X_{2}}\left(D_{F}\right) .
\end{aligned}
$$

When $X_{1}$ and $X_{2}$ are independent equality holds in the last step by definition. Equality in the first line is attainable for all $R_{\text {cache }} \in\left[0, R_{X_{1}}\left(D_{F}\right)+R_{X_{2}}\left(D_{F}\right)\right]$ by splitting the cache message into two parts, one for each decoder. Each decoder then has a personal cache and update link over which the encoder can split the bits of $R_{X_{i}}\left(D_{F}\right)$. Call the size of those parts $\delta_{1}$ and $\delta_{2}$ for decoders 1 and 2 . Let $\delta \in\left[0, R_{X_{1}}\left(D_{F}\right)+\right.$ $R_{X_{2}}\left(D_{F}\right)$. Then $\bar{R}_{\text {update }}$ is minimized by:

$$
\underset{\delta_{1}+\delta_{2}=\delta}{\arg \min } p\left(R_{X_{1}}\left(D_{F}\right)-\delta_{1}\right)^{+}+(1-p)\left(R_{X_{2}}\left(D_{F}\right)-\delta_{2}\right)^{+},
$$

which is solved by distributing rate in a greedy fashion as mentioned in the theorem: the encoder should cache $X_{1}$ exclusively until it satisfies the desired end distortion constraint.

In general settings, the cache message does not have to be decodable on its own. However, if $X_{1}, X_{2}$ are both independent and successively refinable, then caching could also be implemented as an application of successive refinability [20]. Instead of splitting the bits of $R\left(D_{F}\right)$ over both phases, one caches the most popular $X_{i}$ up to some distortion $D_{\text {cache }}$ for $R\left(D_{\text {cache }}\right)$ bits and then refines this to $D_{F} \leq D_{\text {cache }}$ using $R\left(D_{F}\right)-R\left(D_{\text {cache }}\right)$ bits. The cache message in such a setting would be decodable on its own, serving as a 'thumbnail' of what is to come. For Gaussians, this is intrinsic to $R_{\text {cache }}\left(d, D_{F}\right)$ (18).

\section{DEPENDENCE, BUT NO PREFERENCE}

If it is equally likely that the user picks $X_{1}$ or $X_{2}$ then preference cannot be leveraged. If, however, the sources are dependent then the encoder could cache the information that is shared by both files; after all, that shared information is useful no matter the choice the user makes, whereas information that is unique to one file might go to waste. We will argue that if $p=\frac{1}{2}$ the caching problem becomes an application of the concepts of Wyner's common information [9] and Watanabe's total correlation [10]. In a Gaussian setting, these concepts are encapsulated in the Hadamard inequality. The consequence is that symmetry in user preference results in symmetry in the caching strategy:

Theorem 2. If $p=1-p=\frac{1}{2}$, then the Gaussian boundary of the caching rate-distortion region is characterized by

$$
\begin{aligned}
R_{\text {cache }}\left(d, D_{F}\right) & =R(d, d) \\
& = \begin{cases}\frac{1}{2} \log \frac{1-\rho^{2}}{d^{2}} & d \in\left[D_{F}, 1-|\rho|\right], \\
\frac{1}{2} \log \frac{1-\rho^{2}}{d^{2}-(d-(1-|\rho|))^{2}} & d \in[1-|\rho|, 1],\end{cases}
\end{aligned}
$$

for a normalized parameter $d \in\left[D_{F}, 1\right]$ that relates back to $\bar{R}_{\text {update }}$ by picking $d=D_{F} 2^{2 \bar{R}_{\text {update }}}$.

The proof will be divided into two key steps, given by upcoming Lemmas 3 and 4. We develop the proof of Theorem 2 in the next subsections at a slower pace than necessary in order to expose these relations to common information and total correlation. The result is also plotted in Figure 5.

\section{A. Efficient Gaussian Caching under Uniform Preference is Closing the Hadamard Inequality}

In Corollary 1 we established that a Gaussian caching strategy can be picked by means of an MSE distortion matrix $\mathbf{D}$ and that without loss of optimality one can always pick a matrix that is rate-distortion optimal with respect to $R\left(D_{1}, D_{2}\right)$. Instead of optimizing over all matrices $\mathbf{D}$, one would then only have to optimize over the marginal distortions $D_{1}$ and $D_{2}$. Let us, however, take one step back and evaluate ( $\left.R_{\text {cache }}, \bar{R}_{\text {update }}\right)$ of a general Gaussian strategy:

$$
\left\{\begin{array}{l}
R_{\text {cache }}=\frac{1}{2} \log \frac{\left|\Sigma_{\mathbf{X}}\right|}{|\mathbf{D}|}, \\
\bar{R}_{\text {update }}=\frac{1}{2} \log \frac{D_{1,1}^{1 / 2} D_{2,2}^{1 / 2}}{D_{F}}=\frac{1}{4} \log \frac{D_{1,1} D_{2,2}}{D_{F}^{2}} .
\end{array}\right.
$$

Small $R_{\text {cache }}$ requires the determinant to be large, whereas a small $\bar{R}_{\text {update }}$ forces us to minimize the product of the diagonal entries of $\mathbf{D}$. These two are related in the Hadamard inequality

$$
D_{1,1} D_{2,2} \geq|\mathbf{D}|
$$

which relates to a classic information theoretic inequality

$$
h\left(X_{1} \mid V\right)+h\left(X_{2} \mid V\right) \geq h(\mathbf{X} \mid V),
$$

by realizing the following relationship:

$$
\begin{cases}h\left(X_{1} \mid V\right)+h\left(X_{2} \mid V\right) & =\frac{1}{2} \log (2 \pi e)^{2} D_{1,1} D_{2,2}, \\ h(\mathbf{X} \mid V) & =\frac{1}{2} \log (2 \pi e)^{2}|\mathbf{D}| .\end{cases}
$$

A review on this one-to-one correspondence can be read in, e.g., the book by Cover and Thomas [21, Section 17.9]. These inequalities put the following limit on performance:

Lemma 2. The Gaussian caching rate-distortion function is lower bounded as:

$$
R_{\text {cache }}\left(d, D_{F}\right) \geq \frac{1}{2} \log \frac{\left|\Sigma_{\mathbf{X}}\right|}{d^{2}}
$$




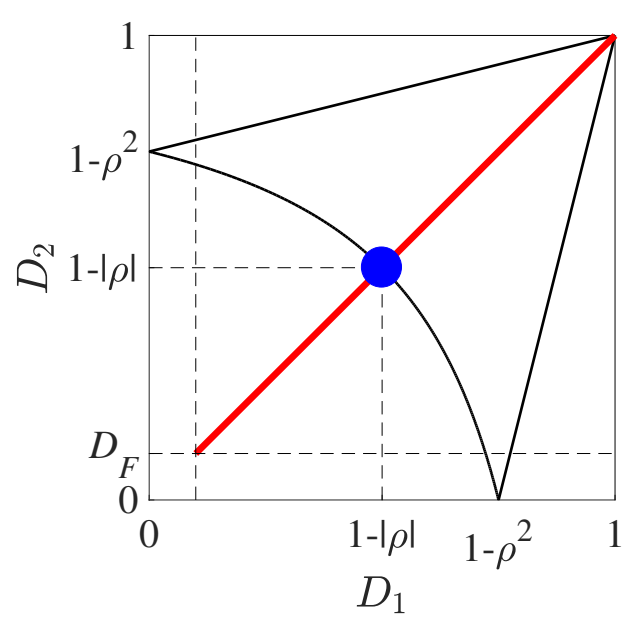

(a) Optimal caching strategies require $D_{1}=D_{2}$.

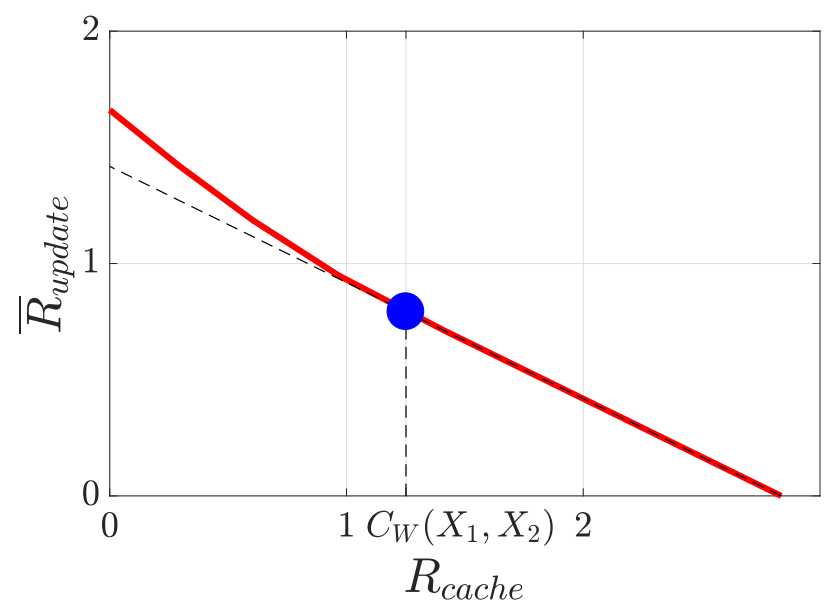

(b) Example of the consequent $\left(R_{\text {cache }}, \bar{R}_{\text {update }}\right)$ trade-off.

Fig. 5: If $p=\frac{1}{2}$, optimal caching strategies lie on the diagonal in the $\mathcal{D}$-plane. The blue dot corresponds to $R_{\text {cache }}=C_{W}\left(X_{1}, X_{2}\right)$ (34). For illustrative purposes, the left is drawn with $\rho=0.5$ and the right with $\rho=0.7$. $D_{F}=0.1$.

Proof. The determinant is bounded by the product of the diagonal, which in turn is bounded by the constraint on $\bar{R}_{\text {update }}$ (by the definition of $R_{\text {cache }}\left(d, D_{F}\right)$ ):

$$
d^{2} \geq D_{1,1} D_{2,2} \geq|\mathbf{D}|
$$

Realizing that $R_{\text {cache }}$ is minimized by maximizing $|\mathbf{D}|$, the encoder must find a distortion profile $\mathbf{D}$ that closes the gap on the Hadamard inequality. Reflecting on the information theoretic version of the inequality: a good caching strategy is one that exploits as much of the dependence as possible in the cache phase, such that as little as possible goes to waste in the individual update. Whether equality is attainable or not separates the caching problem into two distinct regions.

\section{B. High Cache Rate Region \& Common Information}

Equality on the Hadamard inequality requires the caching distortion profile $\mathbf{D}$ to be diagonal. For Gaussians this implies

$$
h\left(X_{1} \mid V\right)+h\left(X_{2} \mid V\right)=h(\mathbf{X} \mid V),
$$

$X_{1}$ and $X_{2}$ have to become conditionally independent given $V$. This is not trivially attainable. Namely, if $\mathbf{D}$ must be diagonal, then $|\mathbf{D}|$ cannot be arbitrarily large in order for $\mathbf{D} \preceq \Sigma_{\mathbf{X}}$ to hold. From an information theoretic perspective, to make $X_{1}$ and $X_{2}$ conditionally independent, $I(\mathbf{X} ; V)$ cannot be arbitrarily small. The latter formulation led to the concept of Wyner's Common Information:

Definition 2 ([9]). Wyner's Common Information equals

$$
C_{W}\left(X_{1}, X_{2}\right) \triangleq \min _{X_{1}-V-X_{2}} I(\mathbf{X} ; V)
$$

Thanks to [22] one knows that for two Gaussians

$$
C_{W}\left(X_{1}, X_{2}\right)=\frac{1}{2} \log \frac{1-|\rho|}{1+|\rho|},
$$

which corresponds to a jointly Gaussian $V$ which results in the following distortion

$$
\mathbf{D}_{C_{W}}=\left[\begin{array}{cc}
1-|\rho| & 0 \\
0 & 1-|\rho|
\end{array}\right]
$$

$C_{W}\left(X_{1}, X_{2}\right)$ being a minimum has consequences on the ( $\left.R_{\text {cache }}, \bar{R}_{\text {update }}\right)$ trade-off as follows:

Lemma 3. Equality in Lemma 2 is achievable if and only if $R_{\text {cache }}\left(d, D_{F}\right) \geq C_{W}\left(X_{1}, X_{2}\right)$, which is in the following range of $d$ :

$$
d \in \begin{cases}{\left[D_{F}, 1-|\rho|\right]} & \text { if } D_{F} \leq 1-|\rho| \\ \emptyset & \text { if } D_{F}>1-|\rho|\end{cases}
$$

Proof. Tying this and the previous section together: Equality in Lemma 2 requires equality in (33), which for Gaussians means that $X_{1}$ and $X_{2}$ become conditionally independent. By the definition of Wyner's Common Information, this can only be done if $R_{\text {cache }} \geq C_{W}\left(X_{1}, X_{2}\right)$.

For $D_{F}>1-|\rho|$, however, $R\left(D_{F}, D_{F}\right)<C_{W}\left(X_{1}, X_{2}\right)$. Therefore, even if the encoder were to cache $X_{1}$ and $X_{2}$ together and completely it would not need more rate than the common information. It is the regime where $\left(D_{F}, D_{F}\right) \in \mathcal{D}_{2}$ (as defined in (7)). For $D_{F} \leq 1-|\rho|$, the lower bound is achievable for all $R_{\text {cache }} \in\left[C_{W}\left(X_{1}, X_{2}\right), R\left(D_{F}, D_{F}\right)\right]$. For example, let $\alpha \in\left[\frac{D_{F}}{1-|\rho|}, 1\right]$ and construct $\mathbf{D}^{\prime}=\alpha \mathbf{D}_{C_{W}}$. Then it holds that $\mathbf{D}^{\prime} \preceq \mathbf{D}_{C_{W}} \preceq \Sigma_{\mathbf{X}}$ (it is an achievable Gaussian distortion matrix) and all $\mathbf{D}^{\prime}$ are diagonal, achieving the optimum and spanning all $R_{\text {cache }} \in\left[C_{W}\left(X_{1}, X_{2}\right), R\left(D_{F}, D_{F}\right)\right]$.

Corollary 2. If $R_{\text {cache }}\left(d, D_{F}\right) \geq C_{W}\left(X_{1}, X_{2}\right)$ then the Gaussian caching rate-distortion function $R_{\text {cache }}\left(d, D_{F}\right)$ also characterizes the boundary of $\mathcal{R}_{\text {caching }}$ in general. 
Proof. This optimality of Gaussian auxiliaries is a consequence of the rate-distortion function being separable over all links of the Gray-Wyner network:

$$
\begin{aligned}
R_{\text {cache }}+R_{u, 1}+R_{u, 2} & =\frac{1}{2} \log \frac{\left|\Sigma_{\mathbf{X}}\right|}{|\mathbf{D}|}+\frac{1}{2} \log \frac{D_{1}}{D_{F}}+\frac{1}{2} \log \frac{D_{2}}{D_{F}} \\
& =\frac{1}{2} \log \frac{1-\rho^{2}}{D_{1} D_{2}}+\frac{1}{2} \log \frac{D_{1} D_{2}}{D_{F}^{2}} \\
& =\frac{1}{2} \log \frac{1-\rho^{2}}{D_{F}^{2}} \\
& =R\left(D_{F}, D_{F}\right) .
\end{aligned}
$$

Evidently, one cannot do better than the joint rate-distortion function. In this regime caching achieves the third outer bound described in Section III-C.

The full story of the separability of $R\left(D_{1}, D_{2}\right)$ on the GrayWyner network and the role of Wyner's Common Information can be found in the work of Viswanatha, Akyol and Rose [11].

Corollary 3. If $R_{\text {cache }} \geq C_{W}\left(X_{1}, X_{2}\right)$ then there exist infinitely many distortion profiles $\mathbf{D}$ that optimize $R_{\text {cache }}\left(d, D_{F}\right)$.

Proof. For $\left(D_{1}, D_{2}\right) \in \mathcal{D}_{1}$ (6) the joint rate-distortion function behaves as $R\left(D_{1}, D_{2}\right)=\frac{1}{2} \log \frac{1-\rho^{2}}{D_{1} D_{2}}$. Consequently the cache and update phase fit together seamlessly for caching strategies inside $\mathcal{D}_{1}$, since $\bar{R}_{\text {update }}=\frac{1}{4} \log \frac{D_{1} D_{2}}{D_{F}^{2}}$ (also mentioned in the previous corollary). Hence, if $d \leq 1-|\rho|$ then any $\mathbf{D}=\operatorname{diag}\left(D_{1}, D_{2}\right)$ of which $D_{1} D_{2}=d^{2}$ and $\left(D_{1}, D_{2}\right) \in \mathcal{D}_{1}$ constitute the same performance of $\left(R_{\text {cache }}, \bar{R}_{\text {update }}\right)$. In other words, for $R_{\text {cache }} \geq C_{W}\left(X_{1}, X_{2}\right)$ infinitely many optimal caching strategies lie in $\mathcal{D}_{1}$ on hyperbola $\frac{1}{D_{1}, D_{2}}=\frac{1}{d^{2}}$ as depicted in Figure 6.

The high cache rate regime is plotted on the right-hand side of Figure $5 \mathrm{~b}$. Observe how the slope of the $\left(R_{\text {cache }}, \bar{R}_{\text {update }}\right)$ trade-off becomes constant. This is the result of the caching strategy capturing all the information that is shared between $X_{1}$ and $X_{2}$. For $R_{\text {cache }} \geq C_{W}\left(X_{1}, X_{2}\right)$ all that remains to cache is information that is individual to either $X_{i}$. Therefore, should one decide to continue caching then only a per-file gain remains.

\section{Low Cache Rate Region \& Total Conditional Correlation}

The previous section introduced the notion of Wyner's Common Information. It was associated to a $R_{\text {cache }}$ large enough to carry all the information that is shared between $X_{1}$ and $X_{2}$. In the region $R_{\text {cache }}<C_{W}\left(X_{1}, X_{2}\right)$ it must be so that for any caching auxiliary $V$ it holds that (31) is strict:

$$
I\left(X_{1} ; X_{2} \mid V\right)=h\left(X_{1} \mid V\right)+h\left(X_{2} \mid V\right)-h(\mathbf{X} \mid V)>0 .
$$

This brings us to a notion that is closely related to common information:

Definition 3. [10] Let $\mathbf{X}$ be a $K$-dimensional random variable. Then Watanabe's total correlation equals

$$
T C(\mathbf{X})=\sum_{i=1}^{K} h\left(X_{i}\right)-h(\mathbf{X}),
$$

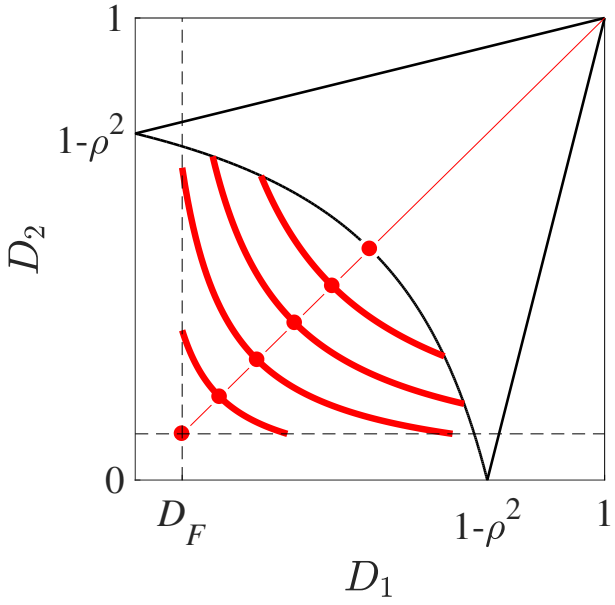

Fig. 6: Once $R_{\text {cache }}\left(d, D_{F}\right) \geq C_{W}\left(X_{1}, X_{2}\right)$, the optimal caching strategies lie inside $\mathcal{D}_{1}$ where the joint rate-distortion function is separable. Therefore, all caching strategies on the hyperbola $\frac{1}{D_{1} D_{2}}=\frac{1}{d^{2}}$ achieve $R_{\text {cache }}\left(d, D_{F}\right)$.

which extends to total conditional correlation as:

$$
T C(\mathbf{X} \mid V)=\sum_{i=1}^{K} h\left(X_{i} \mid V\right)-h(\mathbf{X} \mid V) .
$$

In our bivariate case this expression simplifies to $I\left(X_{1} ; X_{2} \mid V\right)$, but we will refer to it as total conditional correlation nonetheless. In this terminology, the insights so far can be restated as saying that when $R_{\text {cache }} \geq C_{W}\left(X_{1}, X_{2}\right)$ we can apply caching strategies for which $T C(\mathbf{X} \mid V)=0$ no shared information is left after caching.

For $R_{\text {cache }}<C_{W}\left(X_{1}, X_{2}\right)$, whatever cache auxiliary $V$ one picks it must be so that $T C(\mathbf{X} \mid V)>0$, but the encoder should try to get as close to 0 as possible. One thing is certain for low cache rates: $\mathbf{D} \preceq \Sigma_{\mathbf{X}}$ is an active constraint (it is not a strict inequality). Namely, if it were not active then there would be opportunity to get closer to the bound of Lemma 2 until it is ${ }^{1}$. Consequently, if $\mathbf{D}^{*}$ is the minimizer of (18) then it must be that

$$
\operatorname{dim}\left(\operatorname{ker}\left(\Sigma_{\mathbf{X}}-\mathbf{D}^{*}\right)\right) \neq 0 .
$$

Combining this with the condition $\mathbf{D} \preceq \Sigma_{\mathbf{X}}$, the optimal distortion profile takes on the form

$$
\mathbf{D}^{*}=\Sigma_{\mathbf{X}}-\mathbf{v} \mathbf{v}^{T} \succeq 0 ;
$$

correlation is minimized by a rank-one correction along some subspace of $\Sigma_{\mathbf{X}}$. The question is: which?

Lemma 4. Assume w.l.o.g. $\rho>0$, then for all $d \geq 1-|\rho|$, $R_{\text {cache }}\left(d, D_{F}\right)$ is optimized for the following distortion matrix:

$$
\mathbf{D}=\Sigma_{\mathbf{X}}-(1-d) \mathbf{1 1}^{T} .
$$

Proof. Having established that $\mathbf{D} \preceq \Sigma_{\mathbf{X}}$ must be an active constraint, conclude that $\Sigma_{\mathbf{X}}-\mathbf{D}$ is singular and that therefore

\footnotetext{
${ }^{1}$ One trick would be rotation: Given a caching strategy $\mathbf{D}$, multiply this distortion with a rotation matrix. The determinant (and hence $R_{\text {cache }}$ ) remains constant, while the product of the diagonal entries (hence $R_{\text {update }}$ ) can improve. One can keep rotating until $\mathbf{D} \prec \Sigma_{\mathbf{X}}$ becomes $\preceq$.
} 


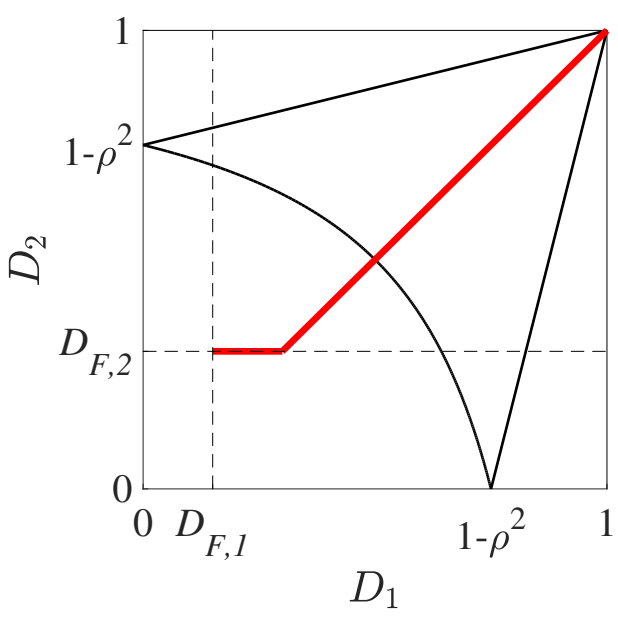

Fig. 7: Optimal caching strategies when using separate end distortion constraints on both files (Corollary 4).

the optimal distortion profile can be modeled as a rank-one correction $\mathbf{D}=\Sigma_{\mathbf{X}}-\alpha \mathbf{v v}^{T}$, for some normalized vector $\mathbf{v}$ and scalar $\alpha$. Let us maximize the determinant of this expression, as that is the objective of $R_{\text {cache }}\left(d, D_{F}\right)$ :

$$
\begin{aligned}
R_{\text {cache }}\left(d, D_{F}\right) \rightarrow & \max _{\mathbf{D}}|\mathbf{D}| \\
& =\max _{\alpha, \mathbf{v}}\left(1-\alpha v_{1}^{2}\right)\left(1-\alpha v_{2}^{2}\right)-\left(\rho-\alpha v_{1} v_{2}\right)^{2} \\
& \leq \max _{\alpha, \mathbf{v}} d^{2}-\left(\rho-\alpha v_{1} v_{2}\right)^{2} .
\end{aligned}
$$

The last step follows from the constraint $D_{1,1}^{1 / 2} D_{2,2}^{1 / 2}=d$. Let us continue by finding the argument that maximizes the above:

$$
\underset{\alpha, \mathbf{v}}{\arg \min }\left(\rho-\alpha v_{1} v_{2}\right)^{2}=\underset{\alpha, \mathbf{v}}{\arg \max } \alpha v_{1} v_{2}
$$

Assume w.l.o.g. that $\left\{v_{1}, v_{2}\right\}$ are normalized ( $\alpha$ can take care of proper scaling). Then we can add the constraint that $v_{1}^{2}+v_{2}^{2}=1$. We thus end up at maximizing a product of numbers under a sum constraint, which is known to be solved by taking all numbers equal. Thus, $v_{1}=v_{2}=\frac{1}{\sqrt{2}}$. Plugging in $\left(1-\alpha v_{1}^{2}\right)\left(1-\alpha v_{2}^{2}\right)=d$ from (40) tells us that $\alpha=2(1-d)$, and thus

$$
\mathbf{D}=\left[\begin{array}{ll}
1 & \rho \\
\rho & 1
\end{array}\right]-(1-d) \mathbf{1 1}^{T}
$$

The above holds for $\rho>0$. Otherwise, one can verify that $v_{1}=-v_{2}$ is the right solution. Both cases combined gives us $|\mathbf{D}|=d-(|\rho|-(1-d))^{2}$.

On a side note, this distortion matrix (41) is inevitably also the optimizer of the joint-rate distortion function subject to symmetric distortions $R(d, d)$; the insight of the proof is thus more that indeed these marginal distortions need to be equal for caching to be optimal. Observe as well that the all-ones vector in the rank-one correction is the dominant eigenvector of the correlation matrix $\Sigma_{\mathbf{X}}$ (for $\rho>0$ ).

Lemma 3 and 4 together constitute Theorem 2 and Figure 5. We emphasize the nuance that the caching strategy in this low cache rate regime is unique, whereas it is not in the high rate regime (by Corollary 3 ).
D. Variance is irrelevant - Reverse Water-Filling the Correlation Matrix

1) Asymmetric distortion constraints: Setting the end distortion constraint $D_{F}$ the same for both files results in an optimal caching strategy (in this scenario when $p=1-p$ ) in which $X_{1}$ and $X_{2}$ reach the end distortion criteria simultaneously when $R_{\text {cache }}=R\left(D_{F}, D_{F}\right)$. The impact of choosing individual criteria for both sources leaves Theorem 2 intact for all $R_{\text {cache }}$ up to the point where either $\hat{X}_{i}$ reaches its end criterion first. Let $D_{F, 1}$ (resp. $D_{F, 2}$ ) be the end distortion constraint for $\hat{X}_{1}\left(\right.$ resp. $\left.\hat{X}_{2}\right)$. Define $R_{\text {cache }}\left(d,\left[D_{F, 1} D_{F, 2}\right]\right)$ to be the cache rate-distortion function exactly as (18) but for asymmetric end distortion criteria, valid over $d \in\left[D_{F, 1}^{p} D_{F, 2}^{1-p}, 1\right]$ :

$$
\begin{aligned}
& R_{\text {cache }}\left(d,\left[D_{F, 1} D_{F, 2}\right]\right) \triangleq \min _{\substack{D_{F, 1} \leq D_{1} \leq 1 \\
D_{F, 2} \leq D_{2} \leq 1}} R\left(D_{1}, D_{2}\right) \\
& \text { s.t. } \quad D_{1}^{p} D_{2}^{1-p} \leq d
\end{aligned}
$$

Consider still $p=1-p=\frac{1}{2}$. Then an immediate consequence of the previous subsections is the following corollary, which is also depicted in Figure 7:

Corollary 4. If $D_{F, 1}>D_{F, 2}$, then

$$
R_{\text {cache }}\left(d,\left[D_{F, 1} D_{F, 2}\right]\right)=R\left(\bar{D}_{1}, d^{2} / \bar{D}_{1}\right) \text {, }
$$

where $\bar{D}_{1}=\max \left(d, D_{F, 1}\right)$. If $D_{F, 1}<D_{F, 2}$, switch them.

2) Variance is irrelevant: For the standard problem of Gaussian vector source coding under mean-squared error, the solution can be characterized by what is referred to as a reverse water-filling procedure on the eigenvalues of the covariance matrix [21, Section 10.3.3]. For our setting, in the case of uniform file preference, it is interesting to observe that the solution can also be characterized by a reverse water-filling procedure, but it is on the eigenvalues of the correlation matrix. In other words, the respective variances of the two sources do not matter, only their correlation coefficient does. To see this, let us start by supposing that the covariance matrix of $\left(X_{1}, X_{2}\right)$ is

$$
\Sigma_{\mathbf{X}}=\left[\begin{array}{cc}
\sigma_{1}^{2} & \rho \sigma_{1} \sigma_{2} \\
\rho \sigma_{1} \sigma_{2} & \sigma_{2}^{2}
\end{array}\right] .
$$

As we have seen, with uniform file preference, our problem is characterized by

$$
\max _{\mathbf{0} \preceq \mathbf{D} \preceq \Sigma_{\mathbf{X}}}|\mathbf{D}| \quad \text { s.t. } \quad D_{1,1} D_{2,2} \leq d^{2} .
$$

Also define the matrix $\mathbf{S}=\operatorname{diag}\left(\left[\begin{array}{ll}\sigma_{1} & \sigma_{2}\end{array}\right]\right)$. Then one can decompose $\mathbf{X}=\mathbf{S} \overline{\mathbf{X}}$, of which $\overline{\mathbf{X}}$ are two unit variance Gaussians. Then the optimization above can be equivalently written down as:

$$
\max _{\mathbf{0} \preceq \mathbf{S} \overline{\mathbf{D}} \mathbf{S}^{T} \preceq \mathbf{S} \Sigma_{\overline{\mathbf{X}}} \mathbf{S}^{T}}\left|\mathbf{S} \overline{\mathbf{D}} \mathbf{S}^{T}\right| \quad \text { s.t. } \quad \sigma_{1}^{2} \bar{D}_{1,1} \sigma_{2}^{2} \bar{D}_{2,2} \leq d^{2},
$$

which is solved by the same $\overline{\mathbf{D}}$ as the following normalized version of the problem:

$$
\max _{\mathbf{0} \preceq \overline{\mathbf{D}} \preceq \Sigma_{\overline{\mathbf{x}}}}|\overline{\mathbf{D}}| \quad \text { s.t. } \quad \bar{D}_{1,1} \bar{D}_{2,2} \leq \frac{d^{2}}{\sigma_{1}^{2} \sigma_{2}^{2}} .
$$

Hence, evidently, only the correlation coefficient between the sources matters; the variances $\sigma_{1}^{2}$ and $\sigma_{2}^{2}$ are irrelevant. 


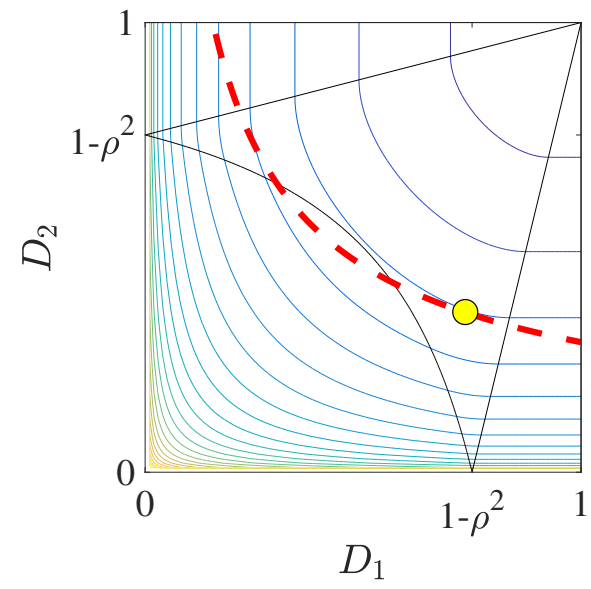

(a) The optimal caching strategy is where a contour line of $R\left(D_{1}, D_{2}\right)$ is tangential to the (dashed) line $D_{1}^{p} D_{2}^{1-p}=d$.

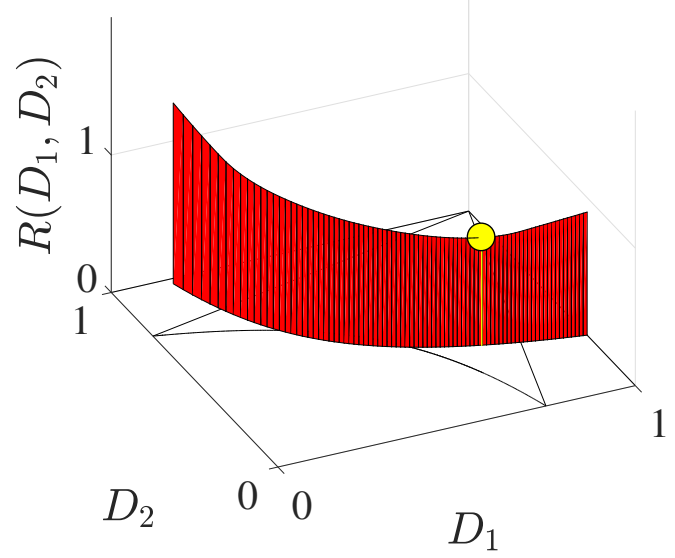

(b) A slice of $R\left(D_{1}, D_{2}\right)$ that is strictly convex in $\left(D_{1}, D_{2}\right)$ has one unique minimum.

Fig. 8: Example of Lemma 5 for $p=\frac{2}{5}$ and $d=0.475$.

\section{The Full Picture: Dependent Sources with Non-Uniform PrefEREnce Probabilities}

This section discusses the full $\left(R_{\text {cache }}, \bar{R}_{\text {update }}\right)$ trade-off by considering $0<|\rho|<1$ and arbitrary $p$. So far the following cases are understood:

- Section IV, $\rho=0$ (or $p \in\{0,1\}$ ):

Cache the most popular $X_{i}$ first and exclusively,

i.e., $\left(D_{1}, D_{2}\right)$ must lie on the border of $\mathcal{D}_{2}$ and $\mathcal{D}_{3}$.

- Section $\mathrm{V}, p=\frac{1}{2}$ :

Cache $X_{1}$ and $X_{2}$ equally,

i.e., $\left(D_{1}, D_{2}\right)$ must lie on the diagonal of the $\mathcal{D}$-plane.

In this section we argue that for any $p$ between those extremes, the optimal caching strategy is a distortion pair $\left(D_{1}, D_{2}\right)$ that lies between the diagonal and the border of $\mathcal{D}_{2}$ and $\mathcal{D}_{3}$.

The optimal $\left(R_{\text {cache }}, \bar{R}_{\text {update }}\right)$ trade-off and the strategies that attain it are shown by example in Figure 9. These curves are derived in the next two pages. To the best of our knowledge, no closed-form analytic expression exists for expressing these optimal cache-distortions in terms of $p, \rho$ and $d$. Numerically, however, the problem is not hard.

First of all, it should be clear that if $p<1-p$ then a good caching strategy should mostly cache information on $X_{2}$ and must thus result in $D_{1}>D_{2}$. To that end, let us cut up the $\mathcal{D}$-plane in an upper and lower triangle:

$$
\begin{aligned}
& \mathcal{D}_{i, 1}=\mathcal{D}_{i} \cap\left\{D_{1}, D_{2}: D_{2} \geq D_{1}\right\}, \\
& \mathcal{D}_{i, 2}=\mathcal{D}_{i} \cap\left\{D_{1}, D_{2}: D_{2} \leq D_{1}\right\} .
\end{aligned}
$$

Lemma 5. If $D_{F}=0$, the cache-update trade-off has one unique minimum on the $\mathcal{D}$-plane, which is the solution to

$$
R_{\text {cache }}(d, 0)=\min _{D_{1}} R\left(D_{1}, d^{\frac{1}{1-p}} D_{1}^{-\frac{p}{1-p}}\right) .
$$

Proof. Neglect the constraint involving $D_{F}$. In (18) one does not evaluate $R\left(D_{1}, D_{2}\right)$ over all $\left(D_{1}, D_{2}\right) \in \mathcal{D}$, but only along a 'slice' defined by the constraint:

$$
D_{1}^{p} D_{2}^{1-p}=d \quad \longrightarrow \quad D_{2}=d^{\frac{1}{1-p}} D_{1}^{-\frac{p}{1-p}} .
$$

This slice is strictly convex with respect to $\left(D_{1}, D_{2}\right)$. The contour lines (or isolines) of $R\left(D_{1}, D_{2}\right)$ are also convex (and continuous!) on the $\mathcal{D}$-plane. More importantly, though, these contour lines end straight $\left(\frac{\partial D_{2}}{\partial D_{1}}=0\right.$ in $\mathcal{D}_{3,2}$ and $\frac{\partial D_{1}}{\partial D_{2}}=0$ in $\left.\mathcal{D}_{3,1}\right)$. Consequently, the minimum of $R\left(D_{1}, D_{2}\right)$ evaluated on a strictly convex curve is where that curve is tangential with a contour line; it cannot be at a simple crossing.

This tangential part is either a unique point or a (set of) closed interval(s), the latter if and only if there exists a contour line that is described by the same curve as (45) for some interval(s). This happens in $\mathcal{D}_{1}$ when $p=\frac{1}{2}$, but not for other $p$. Hence, there can only be one minimum.

An illustration of the 'slicing' of Lemma 5 is depicted in Figure 8. Using this as a building block, we end with the following theorem:

Theorem 3. Without loss of generality, assume $p<\frac{1}{2}$. The Gaussian boundary of the caching rate-distortion region is characterized by

$$
R_{\text {cache }}\left(d, D_{F}\right)=R\left(\bar{D}_{1}, d^{\frac{1}{1-p}} \bar{D}_{1}^{-\frac{p}{1-p}}\right)
$$

where $\bar{D}_{1}=\max \left(D_{F}, D_{1}^{*}\right)$ and $D_{1}^{*}$ is the solution to

$$
\begin{aligned}
\frac{\partial}{\partial D_{1}}( & -1+D_{1}+d^{\frac{1}{1-p}} D_{1}^{\frac{-p}{1-p}}+ \\
2 \rho & \left.\sqrt{\left(1-D_{1}\right)\left(1-d^{\frac{1}{1-p}} D_{1}^{\frac{-p}{1-p}}\right)}\right)=0,
\end{aligned}
$$

$\operatorname{over}\left(D_{1}, d^{\frac{1}{1-p}} D_{1}^{-\frac{p}{1-p}}\right) \in \mathcal{D}_{2,2}(44)$.

Proof. First, assume $D_{F}$ plays no restricting role. Then, the minimum of Lemma 5 lies necessarily in $D_{2,2}$. Namely, it 


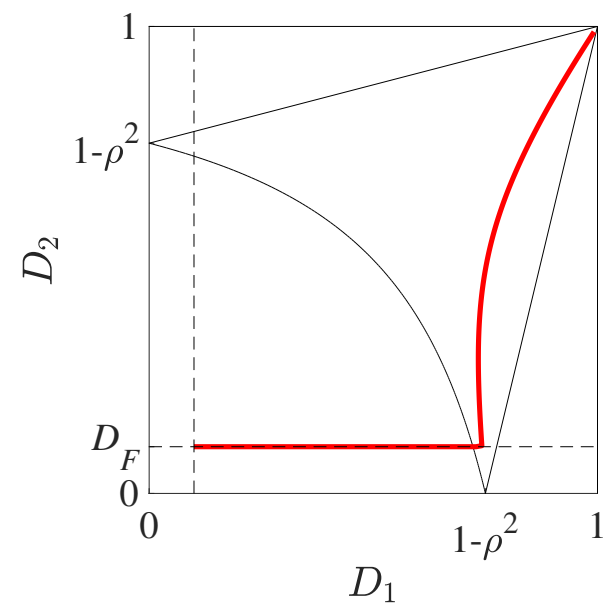

(a) As long as no file is cached completely, optimal strategies lie necessarily in $\mathcal{D}_{2}$.

Fig. 9: Example of optimal caching strategies and performance in terms of $\left(R_{\text {cache }}, \bar{R}_{\text {update }}\right)$ for $p=0.4, \rho=0.5$ and $D_{F}=0.1$. If $D_{F} \leq 1-|\rho|$ then the caching strategies transition from $\mathcal{D}_{2}$ to $\mathcal{D}_{1}$ when $R_{\text {cache }}=R\left(1-\frac{\rho^{2}}{1-D_{F}}, D_{F}\right)$ (see Corollary 5 ).

cannot lie in $\mathcal{D}_{3,2}$ since its boundary is strictly superior. Second, in $\mathcal{D}_{1,2}$ the equipotential lines of $R\left(D_{1}, D_{2}\right)$ behave as $D_{1} D_{2}=$ constant. Hence, they cannot be tangential to the curve $D_{1}^{p} D_{2}^{1-p}=d$, whose derivative is 'less steep' everywhere. That leaves $\mathcal{D}_{2,2}$, where $R\left(D_{1}, D_{2}\right)$ is minimized by maximizing $D_{1} D_{2}-\left(\rho-\sqrt{\left(1-D_{1}\right)\left(1-D_{2}\right)}\right)^{2}$ (5). Вy restricting $\left(D_{1}, D_{2}\right)=\left(D_{1}, d^{\frac{1}{1-p}} D_{1}^{-\frac{p}{1-p}}\right) \in \mathcal{D}_{2,2}$ first and only then setting this derivative w.r.t. $D_{1}$ to 0 , one finds the optimum.

Finally, should any $D_{i}$ drop to $D_{F}$, then $R_{\text {cache }}\left(d, D_{F}\right)$ is minimized at the intersection of $D_{i}=D_{F}$ and $D_{1}^{p} D_{2}^{1-p}=d$, since $R\left(D_{1}, D_{2}\right)$ is monotonic along that curve on one side of the aforementioned unconstrained minimum.

Figure 9 plots an example of Theorem 3. The qualitative interpretation of this result is the following: for $p=\frac{1}{2}$ the optimal strategy was to use all $R_{\text {cache }}$ to make $X_{1}$ and $X_{2}$ conditionally independent as quickly as possible. The opposite is the case if $p \neq 1-p$; after caching there should always remain some dependency between $X_{1}$ and $X_{2}$. The only exception is when one file is cached completely, otherwise the optimal strategy lies necessarily in $\mathcal{D}_{2}$.

Corollary 5. Assume w.l.o.g. that $p<1-p$, then $R_{\text {cache }}\left(d, D_{F}\right)$ also characterizes the boundary of $\mathcal{R}_{\text {caching }}$ in general for $R_{\text {cache }} \geq R\left(1-\frac{\rho^{2}}{1-D_{F}}, D_{F}\right)$, provided $D_{F} \leq 1-|\rho|$.

Proof. For $R_{\text {cache }} \geq R\left(1-\frac{\rho^{2}}{1-D_{F}}, D_{F}\right)$, the optimal caching strategies as described by Theorem 3 lie inside $\mathcal{D}_{1}$. In this region $R\left(D_{1}, D_{2}\right)=\frac{1}{2} \log \frac{1-\rho^{2}}{D_{1} D_{2}} ; X_{1}$ and $X_{2}$ become conditionally independent and the same argument as for Corollary 2 holds. In this high cache rate regime, the Gaussian ( $\left.R_{\text {cache }}, \bar{R}_{\text {update }}\right)$ trade-off attains the outer bound described as number 3 in Subsection III-C.

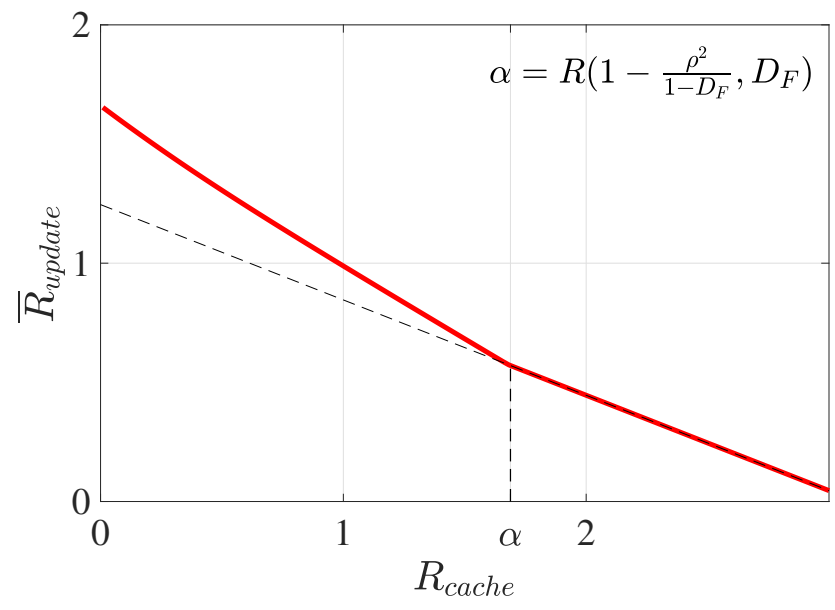

(b) The resulting trade-off between $R_{\text {cache }}$ and $\bar{R}_{\text {update }}$ with bounds. 


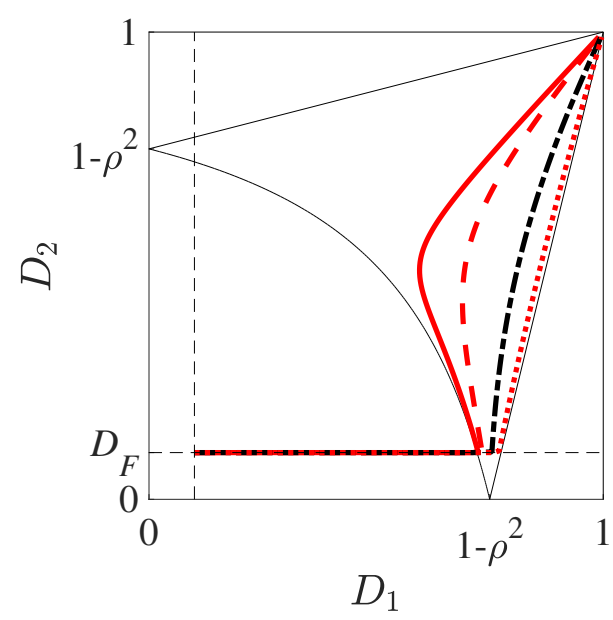

(a) Optimal strategies move away from the diagonal as $p$ decreases.

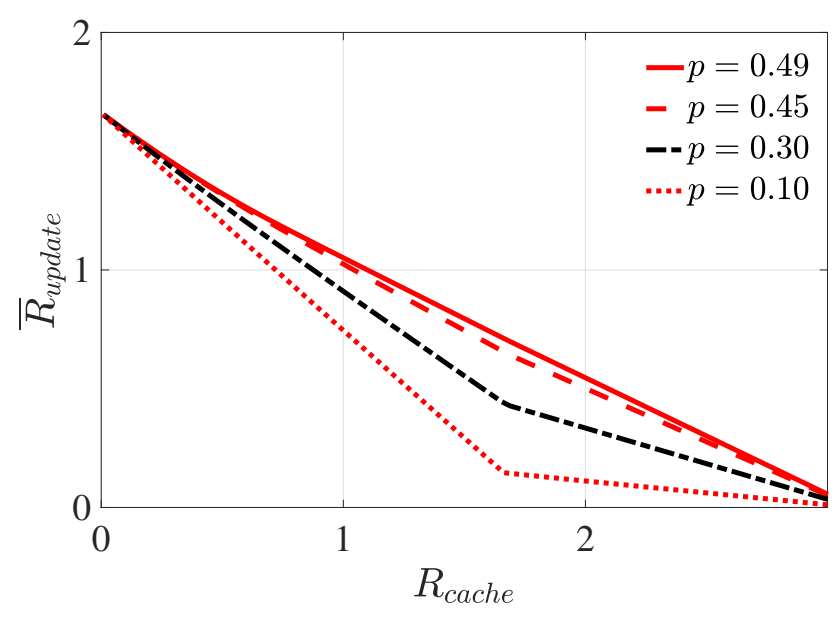

(b) The resulting trade-off between $R_{\text {cache }}$ and $\bar{R}_{\text {update }}$.

Fig. 10: Progression of strategies for $p=0.49,0.45,0.3$ and 0.1 , correlation $\rho=0.5$ and $D_{F}=0.1$.

resulting from that function admit a semidefinite ordering. In particular and tailored towards this work, $R\left(D_{1}, D_{2}\right)$ is successively refinable from $\left(D_{1}^{1}, D_{2}^{1}\right)$ to $\left(D_{1}^{2}, D_{2}^{2}\right) \leq\left(D_{1}^{1}, D_{2}^{1}\right)$ if and only if the resulting distortion matrices respect the order $\mathbf{D}^{1} \preceq \mathbf{D}^{2}$. Nayak et al. furthermore explain how this in $\mathcal{D}_{2}$ requires $\left(D_{1}^{1}, D_{2}^{1}\right)$ and $\left(D_{1}^{2}, D_{2}^{2}\right)$ to lie on a straight line originating from $(1,1)$. Since optimal caching strategies generally lie on a curve instead of a line, it is impossible for the caching phase to be split into two of which both phases are optimal w.r.t. $R_{\text {cache }}\left(d, D_{F}\right)$. The only exceptions are when $p=\frac{1}{2}$ (when optimal strategies lie on the diagonal) or when either $\rho=0$ or $p \in\{0,1\}$ (when the encoder codes only $X_{1}$ or $X_{2}$ ).

A last observation from Figure 10a is that encoder strategies diverge, but eventually converge:

Corollary 7. For $D_{F} \rightarrow 0$ and $R_{\text {cache }} \rightarrow \infty$, the optimal strategy is to only cache the most popular $X_{i}$ irrespective of the exact value $p$.

Proof. $\mathcal{D}_{2}$ ends in two corners, i.e., $\left(0,1-\rho^{2}\right)$ or $\left(1-\rho^{2}, 0\right)$. if $R_{\text {cache }}$ grows very large and $D_{F}$ plays no restricting role, the optimal caching strategy is necessarily squeezed into these corners. These points are associated to a perfect description of one $X_{i}$ and the resulting MSE-estimator of the other. In other words: for very large $R_{\text {cache }}$ the best caching strategy cares more about the most popular component and less about the correlation between the two, irrespective of $p$.

\section{B. Sensitivity to Exact Knowledge of User Preference}

The strong change in optimal strategy as a function of preference begs the question exactly how sensitive caching is to accurate knowledge of the user's habits. Considering the two parameters correlation and preference, one can argue that in practice the encoder most likely has perfect knowledge of the former and only an estimate of the latter.

If the encoder does not know the value of $p$, his best strategy is to assume $p=\frac{1}{2}$. The loss of performance due to this imperfect knowledge is bounded. This is already evident from the geometry of Figure 3: the actual best Gaussian ( $\left.R_{\text {cache }}, \bar{R}_{\text {update }}\right)$ trade-off lies inside the bounded triangle, as well as an encoding strategy of assuming $p=\frac{1}{2}$ (even though that is not the real value of $p$ ). In the following corollary we characterize the maximum loss explicitly. A surprising result is that the maximum loss depends on $p$, but the point where this loss is maximized does not; that only depends on $D_{F}$.

Theorem 4. Assume $D_{F} \leq 1-|\rho|$ and w.l.o.g. that $p<\frac{1}{2}$. Loss due to lack of knowledge of $p$ is no larger than

$$
\Delta_{R_{u}}=\frac{1}{2} \log \left(\frac{1-\frac{\rho^{2}}{1-D_{F}}}{D_{F}}\right)^{\frac{1}{2}-p}
$$

and attains this maximum at

$$
R_{\text {cache }}=R\left(1-\frac{\rho^{2}}{1-D_{F}}, D_{F}\right) .
$$

Proof. Let us use the superscript $\left(R_{\text {cache }}, \bar{R}_{\text {update }}\right)^{\text {actual }}$ to refer to the Gaussian optimal caching trade-off via Theorem 3 based on some fixed $p<\frac{1}{2}$. Introduce as well $\left(R_{\text {cache }}, \bar{R}_{\text {update }}\right)^{\text {unif }}$ to indicate the caching trade-off based on Theorem 2, i.e., when the encoder does not know $p$ and assumes it equals $\frac{1}{2}$. They key is that $\left(R_{\text {cache }}, \bar{R}_{\text {update }}\right)^{\text {actual }}$ and $\left(R_{\text {cache }}, \bar{R}_{\text {update }}\right)^{\text {unif }}$ diverge until $R_{\text {cache }}=R\left(1-\frac{\rho^{2}}{1-D_{F}}, D_{F}\right)$ and then converge. Note that $\left(R_{\text {cache }}, \bar{R}_{\text {update }}\right)^{\text {actual }}$ and $\left(R_{\text {cache }}, \bar{R}_{\text {update }}\right)^{\text {unif }}$ start and end in the same points, $\left(0, \frac{1}{2} \log \frac{1}{D_{F}}\right)$ and $\left(\frac{1}{2} \log \frac{1-\rho^{2}}{D_{F}^{2}}, 0\right)$ respectively.

$\left(R_{\text {cache }}, \bar{R}_{\text {update }}\right)^{\text {unif }}$ is a strictly convex curve on $R_{\text {cache }} \in$ $\left[0, C_{W}\left(X_{1}, X_{2}\right)\right]$, after which it is a straight line connecting $\left(\frac{1}{2} \log \frac{1-|\rho|}{1+|\rho|}, \frac{1}{2} \log \frac{1-|\rho|}{D_{F}}\right)$ to the end $\left(\frac{1}{2} \log \frac{1-\rho^{2}}{D_{F}^{2}}, 0\right)$. The fact that $p \neq \frac{1}{2}$ does not affect performance; $p$ has been made irrelevant because there is complete symmetry in the sources, the end distortion constraints and the caching strategy, i.e., $D_{1}=D_{2}$.

Then, $\left(R_{\text {cache }}, \bar{R}_{\text {update }}\right)^{\text {actual }}$ is a strictly convex curve until Theorem 3 dictates to cache up to distortions lying inside $\mathcal{D}_{1}$. This happens at $R_{\text {cache }}=R\left(1-\frac{\rho^{2}}{1-D_{F}}, D_{F}\right) \geq C_{W}\left(X_{1}, X_{2}\right)$. From that point on, the trade-off is a straight line connecting 


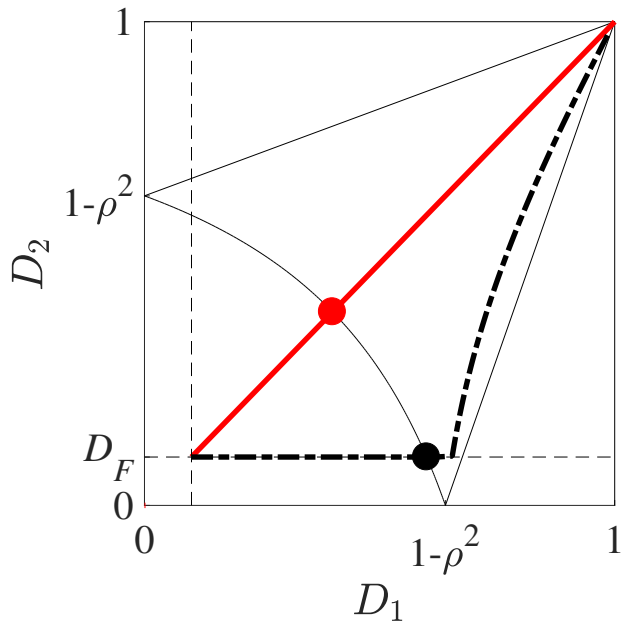

(a) Caching strategies enter $\mathcal{D}_{1}$ at these dots.

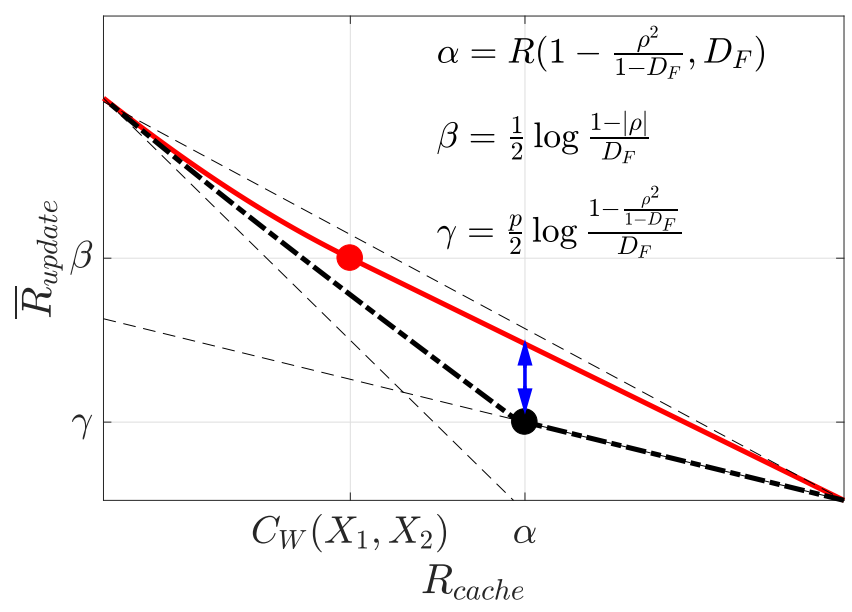

(b) The $\left(R_{\text {cache }}, \bar{R}_{\text {update }}\right)$ trade-offs diverge until both strategies cache up to distortions inside $\mathcal{D}_{1}$; at that point loss is maximal.

Fig. 11: The geometry of how loss due to lack of knowledge of user preference is bounded. Red solid line stands for a uniform caching strategy, whereas the black dotted line stands for what the encoder should do. $p=\frac{1}{4}, \rho=\frac{3}{5}$ and $D_{F}=0.1$.

$\left(\frac{1}{2} \log \frac{1-\rho^{2}}{\left(1-\frac{\rho^{2}}{1-D_{F}}\right) D_{F}}, \frac{p}{2} \log \frac{1-\frac{\rho^{2}}{1-D_{F}}}{D_{F}}\right)$ to the same end point as the uniform strategy.

Because $\left(R_{\text {cache }}, \bar{R}_{\text {update }}\right)^{\text {actual }}$ moves into a straight line at an $R_{\text {cache }}$ strictly larger and an $\bar{R}_{\text {update }}$ strictly smaller than where $\left(R_{\text {cache }}, \bar{R}_{\text {update }}\right)^{\text {unif }}$ makes this change, combined with both curves being strictly convex before reaching that point, must mean that the curves diverge until $R_{\text {cache }}=R\left(1-\frac{\rho^{2}}{1-D_{F}}, D_{F}\right)$. The loss at this point equals

$$
\begin{aligned}
\Delta_{R_{u}} & =\frac{1}{2} \log \frac{\sqrt{\left(1-\frac{\rho^{2}}{1-D_{F}}\right) D_{F}}}{D_{F}}-\frac{p}{2} \log \frac{1-\frac{\rho^{2}}{1-D_{F}}}{D_{F}} \\
& =\frac{1}{2} \log \left(\frac{1-\frac{\rho^{2}}{1-D_{F}}}{D_{F}}\right)^{\frac{1}{2}-p} .
\end{aligned}
$$

The geometry of this loss is depicted in Figure 11.

\section{CACHING FOR $K>2$ FILES}

This last section is devoted to scaling up: to cache databases that contain more than $K=2$ files. The good news is that the general achievable rate region extends quite naturally. The bad news, however, is that it is unclear how exactly the encoder can achieve an optimal operating point inside that rate region.

Before, we identified the two key drivers preference and dependence and derived how the encoder should deal with either one and both together. In higher dimensions, the encoder should deal with preference in the same manner. However, all we derived concerning the impact of dependence among source files does not extend to larger databases as easily. These insights as well as open challenges are the topic of this section.

\section{A. Problem Statement and Model}

Let us extend Section III in the simplest of ways: instead of a database of two sequences of samples $X_{i}^{N}$, the user now has to choose one among $K$, as depicted in Figure 12a. The encoder still transmits one cache message $m_{c}$, but it is followed by one of $K$ possible update messages $m_{u, i}$. Both are still integers between 1 and some number $M_{c}$ or $M_{u, i}$ (for each of the $K$ possible updates). A caching rate-distortion tuple $\left(R_{\text {cache }}, R_{u, 1}, \cdots, R_{u, K}, D_{F}\right)$ is said to be achievable if for arbitrary $\epsilon>0$ there exist encoders and decoders that for both $i=1,2$ we have

$$
\begin{aligned}
M_{c} & \leq 2^{N\left(R_{\text {cache }}+\epsilon\right)} & & \\
M_{u, i} & \leq 2^{N\left(R_{u, i}+\epsilon\right)} & & \text { for } i=1, \cdots, K \\
\frac{1}{N} \sum_{n=1}^{N} d\left(X_{i}(n), \hat{X}_{i}(n)\right) & \leq D_{F}+\epsilon & & \text { for } i=1, \cdots, K
\end{aligned}
$$

where $d_{X}(\cdot, \cdot)$ is some single-letter distortion measure.

As the user now has more to choose from, her choice is captured in the discrete random variable $U$ distributed as $P(U=i)=p_{i}$. Consequently, after caching the encoder expects to still need the following average update rate:

$$
\bar{R}_{\text {update }} \triangleq \sum_{i=1}^{K} p_{i} R_{u, i 1} \text {. }
$$

A shorthand notation will be to say that $\left(R_{\text {cache }}, \bar{R}_{\text {update }}, D_{F}\right)$ is achievable to indicate that (at least one tuple) ( $\left.R_{\text {cache }}, R_{u, 1}, R_{u, 2}, \cdots, R_{u, K}, D_{F}\right)$ is, of which the average update rate equals $\bar{R}_{\text {update }}$.

For the bivariate case a key insight was the analogy to the Gray-Wyner network. That classic scheme can be extended to $K$ sources in numerous ways. For this caching study, the relevant extension would be to again have $K$ decoders (modeling the $K$ choices a user has), connected by one 


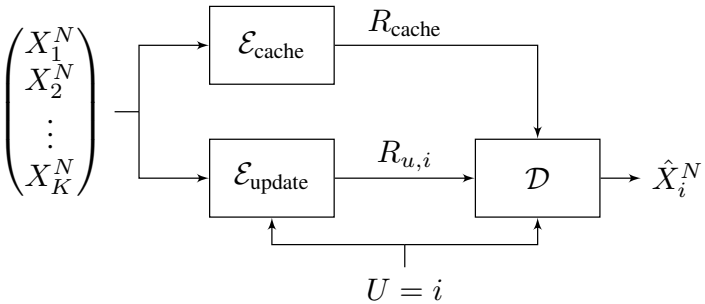

(a) The caching network with a database of $K$ source files.

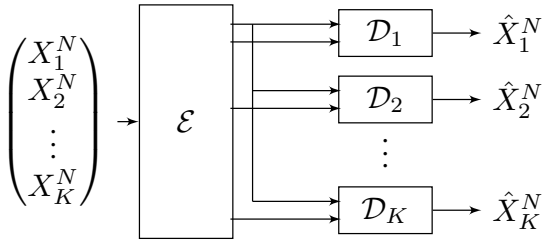

(b) The extended Gray-Wyner network [24].

Fig. 12: The extended caching network for larger databases resembles also the extended Gray-Wyner network.

common link (the cache) and $K$ individual links for each (modeling each possible update message). This extension is drawn in Figure 12b. Its region of achievable communication is also known:

Lemma $6(\mathrm{Xu}, \mathrm{Liu}$ and Chen [24]). Communication on the extended Gray-Wyner network is achievable for rate-distortion tuples $\left(R_{0}, R_{1}, \cdots, R_{K}, D_{1}, D_{2}, \cdots, D_{K}\right)$ that satisfy

$$
\begin{aligned}
& R_{0} \geq I(\mathbf{X} ; \mathbf{V}) \\
& R_{i} \geq R_{X_{i} \mid \mathbf{V}}\left(D_{i}\right) \quad \text { for } i=1,2, \cdots, K,
\end{aligned}
$$

for some conditional distribution $p(\mathbf{v} \mid \mathbf{x})$.

This gives us the following:

Theorem 5. A caching rate-distortion tuple ( $\left.R_{\text {cache }}, \bar{R}_{\text {update }}, D_{F}\right)$ is achievable if and only if there exists $a \mathbf{V}$ and a joint density $p(\mathbf{x}, \mathbf{v})$ such that the following inequalities are satisfied:

$$
\begin{aligned}
R_{\text {cache }} & \geq I(\mathbf{X} ; \mathbf{V}), \\
\bar{R}_{\text {update }} & \geq \sum_{i=1}^{K} p_{i} R_{X_{i} \mid \mathbf{V}}\left(D_{F}\right) .
\end{aligned}
$$

The closure of such achievable tuples over joint densities $p(\mathbf{x}, \mathbf{v})$ is denoted $\mathcal{R}_{\text {caching. }}$

Proof. The extended Gray-Wyner network dictates the achievable rates for the caching problem. Namely, despite the user decoding only one $X_{i}^{N}$, any caching code must have update messages that work for all elements of $\mathbf{X}$ since any one could be requested. The equivalence is that the common link equals the cache and the individual links the update messages for each possible request:

$$
\begin{aligned}
R_{0} & \leftrightarrow R_{\text {cache }}, \\
R_{i} & \leftrightarrow R_{u, i} \quad i=1,2, \cdots, K .
\end{aligned}
$$

Then, take the average over the individual updates as (47).

Now consider specifically a database $\mathbf{X}^{N}$ whose files are sequences of IID samples drawn from a Gaussian distribution with mean $\mathbf{0}$ and covariance

$$
\Sigma_{\mathbf{X}}=\left[\begin{array}{cccc}
1 & \rho_{12} & \rho_{13} & \cdots \\
\rho_{12} & 1 & \rho_{23} & \\
\rho_{13} & \rho_{23} & 1 & \\
\vdots & & & \ddots
\end{array}\right] .
$$

Like the bivariate case, it is unclear whether on the boundary of $\mathcal{R}_{\text {caching }}$ the optimal auxiliary random variable $\mathbf{V}$ would be jointly Gaussian with $\mathbf{X}$. We take this as an assumption and aim to characterize that boundary by restricting to Gaussian codebooks. Later we will prove its optimality in two cases:

1) If the files are independent.

2) If user preference is absent and $R_{\text {cache }}$ is large.

Using these Gaussian codebooks, one can again define a Gaussian caching rate-distortion function. A key difference with the bivariate case, however, is that the Gaussian ratedistortion function subject to individual mean squared errors is not known in closed form (4):

Corollary 8. The Gaussian boundary of $\mathcal{R}_{\text {caching }}$ can be described by the Gaussian caching rate-distortion function:

$$
\begin{aligned}
& R_{\text {cache }}\left(d, D_{F}\right)=\min _{\mathbf{D}} \frac{1}{2} \log \frac{\left|\Sigma_{\mathbf{X}}\right|}{|\mathbf{D}|} \\
& \text { s.t. }\left\{\begin{array}{l}
\mathbf{0} \preceq \mathbf{D} \preceq \Sigma_{\mathbf{X}} \\
\prod_{i=1}^{K} D_{i, i}^{p_{i}} \leq d \\
D_{i, i} \geq D_{F}, \quad \forall i=1,2, \cdots, K
\end{array}\right.
\end{aligned}
$$

for a normalized parameter $d \in\left[D_{F}^{K}, 1\right]$ that relates back to $\bar{R}_{\text {update }}$ by picking $d=D_{F} 2^{2 K \bar{R}_{\text {update }}}$.

Proof. The derivation is analogous to Corollary 1 . The goal is again to express the (Gaussian) boundary of $\mathcal{R}_{\text {caching }}$ by minimizing $R_{\text {cache }}$ while constraining $\bar{R}_{\text {update }}$.

Choosing a $\mathbf{V}$ that is jointly Gaussian with $\mathbf{X}$ in (48) yields:

$$
I(\mathbf{X} ; \mathbf{V})=\frac{1}{2} \log \frac{\left|\Sigma_{\mathbf{X}}\right|}{|\mathbf{D}|},
$$

where again

$$
\mathbf{D}=\mathbb{E}\left[(\mathbf{X}-\mathbb{E}[\mathbf{X} \mid \mathbf{V}])(\mathbf{X}-\mathbb{E}[\mathbf{X} \mid \mathbf{V}])^{T}\right]=\Sigma_{\mathbf{X} \mid \mathbf{V}} \preceq \Sigma_{\mathbf{X}}
$$

Furthermore, $p(\mathbf{x} \mid \mathbf{v})$ being Gaussian results in the update phase for the following:

$$
R_{X_{i} \mid \mathbf{V}}\left(D_{F}\right)=\frac{1}{2} \log +\frac{D_{i, i}}{D_{F}}
$$

Caching any $X_{i}$ beyond $D_{F}$ serves no purpose: it increases $R_{\text {cache }}$ while not improving $R_{u, i}$, since (54) cannot be negative. Therefore, demanding $D_{i, i} \geq D_{F}$ ensures no cache rate is 
wasted on going beyond what is requested. This will thus ensure $R_{u, i} \geq 0$ and hence we arrive at

$$
\begin{aligned}
\bar{R}_{\text {update }} & =\sum_{i=1}^{K} \frac{p_{i}}{2} \log \frac{D_{i, i}}{D_{F}} \\
& =\frac{1}{2} \log \frac{\prod_{i=1}^{K} D_{i, i}^{p_{i}}}{D_{F}} .
\end{aligned}
$$

Combining everything together: Minimizing $R_{\text {cache }}$ over all jointly Gaussian distributions $p(\mathbf{v}, \mathbf{x})$ equals minimizing (52) over distortion matrices $\mathbf{D} \preceq \Sigma_{\mathbf{X}}$. Constraining $\bar{R}_{\text {update }}$ means constraining $\prod_{i=1}^{K} D_{i, i}^{p_{i}}$ for which we introduce a parameter called $d$ to constrain

$$
\prod_{i=1}^{K} D_{i, i}^{p_{i}}=D_{F} 2^{2 K \bar{R}_{\text {update }}} \leq d .
$$

By picking $d \in\left[D_{F}^{K}, 1\right]$, one spans all possible (minimum) values for $\bar{R}_{\text {update }}$, i.e., $\bar{R}_{\text {update }} \in\left[0, \frac{1}{2} \log \frac{1}{D_{F}}\right]$. This gives us the function as defined in the Corollary.

Corollary 8 characterizes thus the boundary of $\mathcal{R}_{\text {caching }}$ when restricting the encoder to pick a caching auxiliary $\mathbf{V}(48)$ that is jointly Gaussian with the database $\mathbf{X}$. Unfortunately, this Gaussian boundary is a non-convex optimization problem. In the following two subsections the function $R_{\text {cache }}\left(d, D_{F}\right)$ is dissected by its two driving parameters: preference and dependence. The main message will be that the latter is the cause of difficulty and that only for large caching rates it can be solved in a convex manner. Consequently, unlike its bivariate counterpart, it is unknown how to solve $R_{\text {cache }}\left(d, D_{F}\right)$ for databases of more than two files.

\section{B. Preference, but no dependence}

By analogy to Section IV, we begin by stating the simple case of independent files, where it is immediately clear that caching proceeds along decreasing file popularity. Specifically, for our Gaussian setting with mean-squared error distortion, we record the following lemma:

Lemma 7. Assume w.l.o.g. that $p_{1} \geq p_{2} \geq \cdots \geq p_{K}$. Define $p_{K+1}=0$. If all $X_{i}$ are independent, then the boundary of $\mathcal{R}_{\text {caching }}$ can be described by

$$
\begin{aligned}
R_{\text {cache }} & =\delta \\
\bar{R}_{\text {update }} & =\sum_{i=1}^{K}\left(p_{i}-p_{i+1}\right) \max \left(0, \sum_{j=1}^{i} R_{X_{i}}\left(D_{F}\right)-\delta\right)
\end{aligned}
$$

Proof. The proof follows the exact same arguments as Theorem 1.

We note that the lossy source coding scenario does not differ in any way from the lossless case [6]. Furthermore, the above holds true for non-Gaussian sources also.

Lastly, even if the source files were dependent, the encoder always has the option to ignore that dependency; the communication rates mentioned above will still be achievable. It is thus more accurate to say that Theorem 7 is an inner bound to the boundary of $\mathcal{R}_{\text {caching, }}$, which is tight if and only if all $X_{i}$ are independent.

\section{Dependence, but no preference}

The difficulty in caching larger databases lies not in user preference, but in how the encoder should capture the dependency among source files. If user preference is absent, then -like in the bivariate case- the encoder should cache the data that minimizes the total conditional correlation. In this section we argue the following asymmetry in caching larger databases: it is easy to identify and cache all the common information between multiple files, but it remains an open problem to identify what part of that common information is most important when one can only cache some.

If $p_{i}=1 / K$, then the product constraint in $R_{\text {cache }}\left(d, D_{F}\right)$ (51) simplifies a little:

$$
\begin{aligned}
R_{\text {cache }}\left(d, D_{F}\right)=\min _{\mathbf{D}} & \frac{1}{2} \log \frac{\left|\Sigma_{\mathbf{X}}\right|}{|\mathbf{D}|} \\
\text { s.t. } & \left\{\begin{array}{l}
\mathbf{0} \preceq \mathbf{D} \preceq \Sigma_{\mathbf{X}} \\
\prod_{i=1}^{K} D_{i, i} \leq d^{K} \\
D_{i, i} \geq D_{F}, \quad \forall i=1,2, \cdots, K
\end{array}\right.
\end{aligned}
$$

To start the analysis, let us recall the definition of total conditional correlation, followed by its simplification (*) if $\mathbf{X}$ and $\mathbf{V}$ are jointly Gaussian with some covariance $\mathbf{D}$ :

$$
\begin{aligned}
T C(\mathbf{X} \mid V) & \triangleq \sum_{i=1}^{K} h\left(X_{i} \mid V\right)-h(\mathbf{X} \mid V) \\
& \stackrel{*}{=} \frac{1}{2} \log \frac{|\mathbf{D}|}{\prod_{i=1}^{K} D_{i, i}}
\end{aligned}
$$

Let the caching rate distortion function be solved also by some matrix D. Like the bivariate case of Section V, the Hadamard inequality implies a bound on caching in the absence of user preference:

$$
\begin{aligned}
R_{\text {cache }}\left(d, D_{F}\right) & =\frac{1}{2} \log \frac{\left|\Sigma_{\mathbf{X}}\right|}{|\mathbf{D}|} \\
& \geq \frac{1}{2} \log \frac{\left|\Sigma_{\mathbf{X}}\right|}{\prod_{i=1}^{K} D_{i, i}} \\
& \geq \frac{1}{2} \log \frac{\left|\Sigma_{\mathbf{X}}\right|}{d^{K}} .
\end{aligned}
$$

Linking this back to (60) we conclude again: a good caching strategy is one that minimizes the total conditional correlation after the caching phase.

1) The Bivariate Caching Strategy is a Bound: In a database of two Gaussian and also neglecting user preference, we derived (specifically in Section V-D) that one can cache the total correlation most efficiently by a reverse water filling procedure on the eigenvalues of the correlation matrix. We will show this is merely an inner bound for higher dimensions. This stems from the inequality of geometric and arithmetic means. To see why, define the following function similar to $R_{\text {cache }}\left(d, D_{F}\right)$, but in which we replace the product constraint by the trace:

$R_{\text {trace }}\left(\gamma, D_{F}\right) \triangleq \min _{\mathbf{D}} \frac{1}{2} \log \frac{\left|\Sigma_{\mathbf{X}}\right|}{|\mathbf{D}|} \quad$ s.t. $\quad\left\{\begin{array}{l}\mathbf{0} \preceq \mathbf{D} \preceq \Sigma_{\mathbf{X}} \\ \operatorname{tr}(\mathbf{D}) \leq \gamma \\ D_{i, i} \geq D_{F}, \quad \forall i .\end{array}\right.$ 


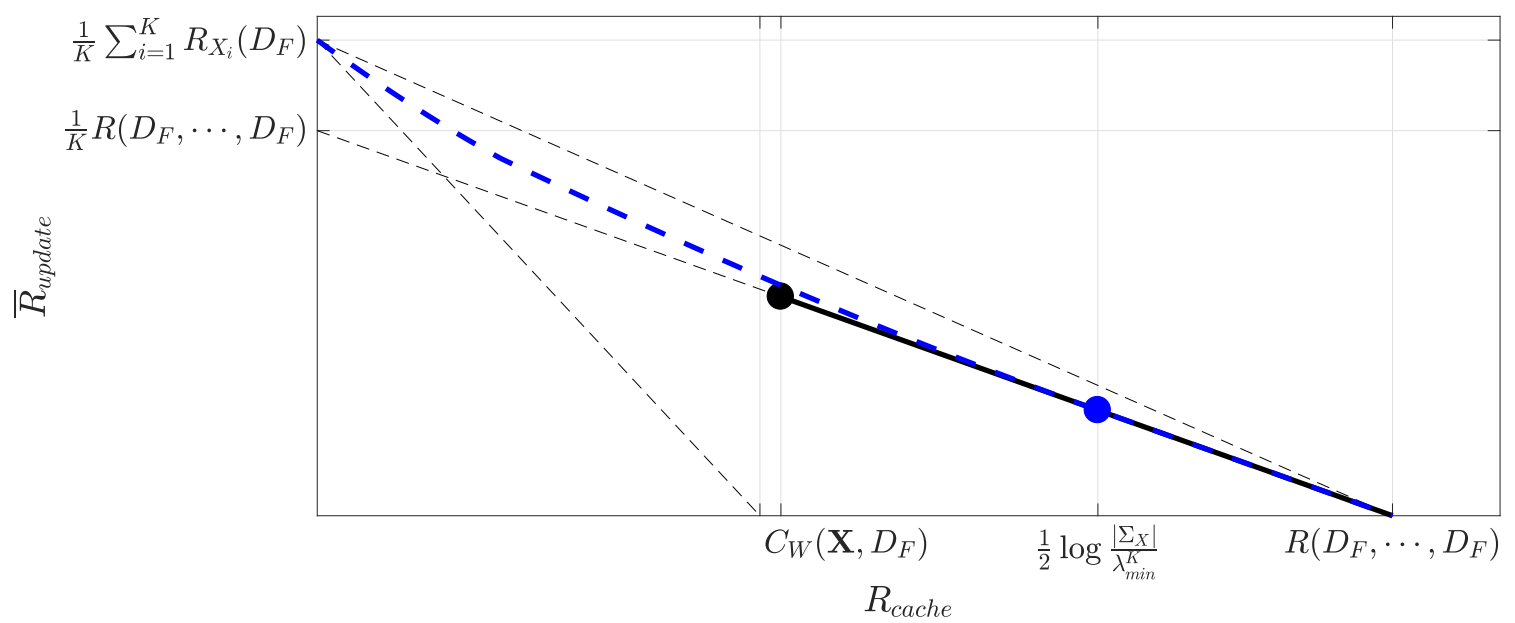

Fig. 13: For large $R_{\text {cache, }}$, Theorem 7 and Corollary 9 state that part of the boundary of $\mathcal{R}_{\text {caching }}$ can be attained by means of Gaussian codebooks by solving a convex optimization problem (thick black line). In general, Lemma 8 shows that a reverse water filling procedure on the eigendecomposition of the correlation matrix as was optimal for $K=2$, merely forms an inner bound if $K>2$ (blue dashed line, representing (64)). Graphics drawn from an example for $K=3$.

Note that $\mathbf{D}$ being a squared error distortion matrix implies that $\operatorname{tr}(\mathbf{D})$ is the sum squared error:

$$
\operatorname{tr}(\mathbf{D})=\sum_{i=1}^{K} \mathbb{E}\left[\left(X_{i}-\mathbb{E}\left[X_{i} \mid \mathbf{V}\right]\right)^{2}\right] .
$$

Lemma 8. The caching rate-distortion function is upper bounded as:

$$
R_{\text {cache }}\left(d, D_{F}\right) \leq R_{\text {trace }}\left(K d, D_{F}\right) .
$$

Proof. The trace of a matrix and the product of its diagonal entries are connected through the inequality of geometric and arithmetic means:

$$
\left(\prod_{i=1}^{K} D_{i, i}\right)^{1 / K} \leq \frac{1}{K} \operatorname{tr}(\mathbf{D}) .
$$

Hence, if we plug in $K d$ then $R_{\text {trace }}\left(K d, D_{F}\right)$ must be solved by a matrix $\mathbf{D}$ of which

$$
\prod_{i=1}^{K} D_{i, i} \leq\left(\frac{1}{K} \operatorname{tr}(\mathbf{D})\right)^{K} \leq d^{K} .
$$

The chain of inequalities imply that the domain of feasible D for $R_{\text {trace }}\left(K d, D_{F}\right)$ is a subset of the search domain of $R_{\text {cache }}\left(d, D_{F}\right)$. Since both functions minimize the same objective function, we must therefore have that $R_{\text {cache }}\left(d, D_{F}\right) \leq$ $R_{\text {trace }}\left(K d, D_{F}\right)$.

The crux of Lemma 8 is the following: if $D_{F}$ is 0 (or more exactly, is so small that it poses no active constraint in (64)), then $R_{\text {trace }}\left(\gamma, D_{F}\right)$ is the 'classic' rate-distortion function of a Gaussian vector source subject to a sum squared error criterion. This function is minimized by a reverse waterfilling procedure on the eigenvalues of the covariance matrix [21, Section 10.3.3]. Now if $\Sigma_{\mathbf{X}}$ is normalized to a correlation matrix, such water-filled distortion matrices were also optimal for caching for $K=2$. The Lemma shows that for $K>2$, though, they merely offer an inner bound. Experiments show that in general one can do caching more efficiently by picking a distortion matrix whose eigenbasis does not commute with the eigenspace of $\Sigma_{\mathbf{X}}$. Figure 13 shows an example of this trace-based inner bound.

2) The High Cache Rate Regime is a Convex Problem: When $R_{\text {cache }}$ is large, figuring out what to cache is -perhaps surprisingly- a convex problem and one can show that Lemma 8 is not tight always. Specifically, the problem of figuring out whether and when equality in (63) is attainable is convex. This will be characterized in Theorem 7. To that end:

Definition 4. We define the multivariate extension of Wyner's common information as

$$
C_{W}(\mathbf{X}) \triangleq \min _{: T C(\mathbf{X} \mid \mathbf{V})=0} I(\mathbf{X} ; \mathbf{V}) .
$$

For discrete random variables a similar extension appeared in [25]. If $\mathbf{X}$ is Gaussian then one can show that the $\mathbf{V}$ that attains $C_{W}(\mathbf{X})$ is necessarily Gaussian as well:

Theorem 6. If $\mathbf{X}$ is Gaussian distributed then the common information equals:

$$
C_{W}(\mathbf{X})=\min _{\mathbf{D}} \frac{1}{2} \log \frac{\left|\Sigma_{\mathbf{X}}\right|}{|\mathbf{D}|} \quad \text { s.t. } \quad\left\{\begin{array}{l}
\mathbf{0} \preceq \mathbf{D} \preceq \Sigma_{\mathbf{X}}, \\
\mathbf{D} \text { is diagonal. }
\end{array}\right.
$$

Proof. The proof uses standard arguments. Consider any $\mathbf{V}$ that is jointly distributed with $\mathbf{X}$ :

$$
\begin{aligned}
I(\mathbf{X} ; \mathbf{V}) & \geq I(\mathbf{X} ; \mathbb{E}[\mathbf{X} \mid \mathbf{V}]) \\
& \geq \frac{1}{2} \log \frac{\left|\Sigma_{\mathbf{X}}\right|}{|\mathbf{D}|} \\
& \geq \frac{1}{2} \log \frac{\left|\Sigma_{\mathbf{X}}\right|}{\prod_{i} D_{i, i}} .
\end{aligned}
$$

The first line follows from the data processing inequality and the second follows from the Gaussian rate distortion function being the lower bound to any $\mathbf{D} \preceq \Sigma_{\mathbf{X}}$ (this inequality is in 
turn implied by using $\mathbb{E}[\mathbf{X} \mid \mathbf{V}]$, see [19, Lemma 2 and 3]). The last line is due to the Hadamard inequality, which is met with equality if and only if $\mathbf{D}$ is diagonal. A diagonal $\mathbf{D}$ stands for zero correlation, which does not guarantee (conditional) independence in general. For Gaussians, however, zero correlation and independence do have an if and only if relationship. Since any distortion matrix $\mathbf{D} \preceq \Sigma_{\mathbf{X}}$ is attainable by Gaussian distributions we can achieve equality throughout all steps.

It is important to note that the constraint of $\mathbf{D}$ having to be diagonal is linear. Hence, the Gaussian common information can always be found efficiently via numerical methods, like the ones described by Boyd and Vandenberghe [26], [27]. Namely, the objective function is strictly convex and the search domain is convex as well. This stands in sharp contrast to the definition of $R_{\text {cache }}\left(d, D_{F}\right)$ in which the constraint $\prod_{i=1}^{K} D_{i, i}^{p_{i}} \leq d$ is nonlinear (even if all $p_{i}=1 / K$ ), which renders that optimization non-convex.

To see how the common information helps one to achieve equality in (63), define again the following:

Definition 5. Let $\mathbf{D}_{C_{W}}$ be the matrix that optimizes (70).

There exist correlation matrices $\Sigma_{\mathbf{X}}$ for which $\mathbf{D}_{C_{W}}$ is very asymmetric, i.e., some diagonal entries are very small in comparison to others. In this regard, one must not forget the other constraint inside $R_{\text {cache }}\left(d, D_{F}\right)$ : the encoder should not cache any $X_{i}$ up to a distortion $D_{i, i}<D_{F}$. A $\mathbf{D}_{C_{W}}$ of which one or some diagonal entries are below $D_{F}$ can therefore never be an optimal caching strategy w.r.t. $R_{\text {cache }}\left(d, D_{F}\right)$. To overcome this, we include this practical coding constraint inside the Gaussian common information:

Definition 6. We define the Gaussian constrained common information to be the following:

$$
C_{W}\left(\mathbf{X}, D_{F}\right)=\inf _{\mathbf{D}} \frac{1}{2} \log \frac{\left|\Sigma_{\mathbf{X}}\right|}{|\mathbf{D}|} \quad \text { s.t. } \quad\left\{\begin{array}{l}
\mathbf{0} \preceq \mathbf{D} \preceq \Sigma_{\mathbf{X}}, \\
\mathbf{D} \text { is diagonal, } \\
D_{i, i} \geq D_{F}, \forall i
\end{array}\right.
$$

In contrast to $C_{W}(\mathbf{X})$, this minimum may not exist. It exists if and only if $\lambda_{\min }\left(\Sigma_{\mathbf{X}}\right) \geq D_{F}$, which is a consequence of combining all three constraints.

If this constrained common information exists, then we have

$$
C_{W}\left(\mathbf{X}, D_{F}\right) \geq C_{W}(\mathbf{X}),
$$

because the search domain of $C_{W}\left(\mathbf{X}, D_{F}\right)$ is a subset of that of $C_{W}(\mathbf{X})$. We note that the constrained common information is also a convex problem, which can be solved by the same numerical methods one would use for (70).

This constrained common information is closely related to the discussion on lossy common information by Viswanatha, Akyol and Rose [11]. They define for two random variables the notion $C_{W}\left(X_{1}, X_{2}, D_{1}, D_{2}\right)$ as the minimum common rate on the Gray-Wyner network needed such that communication of $X_{1}, X_{2}$ is achievable at a sum-rate that does not exceed the joint rate-distortion function $R\left(D_{1}, D_{2}\right)$. This notion was also introduced precisely to cover scenarios where the 'lossless' common information would result in distortions that are more strict than desired by the lossy coding application. Especially insightful is that Viswanatha et al. explicitly characterize the bivariate Gaussian case of their measure.

Using this new constrained $C_{W}\left(\mathbf{X}, D_{F}\right)$, the high cache rate regime as referred to by the title of this subsection relates to the following theorem:

Theorem 7. If $C_{W}\left(\mathbf{X}, D_{F}\right)$ exists, then for all $d \leq\left|\Sigma_{\mathbf{X}}\right| 2^{-\frac{2}{K} C_{W}\left(\mathbf{X}, D_{F}\right)}$ it holds that

$$
R_{\text {cache }}\left(d, D_{F}\right)=\frac{1}{2} \log \frac{\left|\Sigma_{\mathbf{X}}\right|}{d^{K}} .
$$

If furthermore $D_{F} \leq \min \left(\operatorname{diag}\left(\mathbf{D}_{C_{W}}\right)\right)$, then the above holds for all $d \leq\left|\Sigma_{\mathbf{X}}\right| 2^{-\frac{2}{K}} C_{W}(\mathbf{X})$.

Proof. As stated in its definition, $C_{W}\left(\mathbf{X}, D_{F}\right)$ exists if and only if $\lambda_{\min }\left(\Sigma_{\mathbf{X}}\right) \geq D_{F}$. This conditions also implicates equality of the following three expressions:

$$
R\left(D_{F}, \cdots, D_{F}\right)=R_{\text {cache }}\left(D_{F}^{K}, D_{F}\right)=\frac{1}{2} \log \frac{\left|\Sigma_{\mathbf{X}}\right|}{D_{F}^{K}} .
$$

The joint rate-distortion function $R\left(D_{F}, \cdots, D_{F}\right)$ is solved by the diagonal matrix $D_{F} \cdot \mathbf{I} \preceq \Sigma_{\mathbf{X}}$ [19, Lemma 4] and this matrix also is the solution to $R_{\text {cache }}\left(d, D_{F}\right)$ corresponding to the operating point $\left(R_{\text {cache }}, \bar{R}_{\text {update }}\right)=\left(R\left(D_{F}, \cdots, D_{F}\right), 0\right)$.

Then, since $C_{W}\left(\mathbf{X}, D_{F}\right)$ is a minimum, a diagonal $\mathbf{D}$ and hence equality on (63) is not attainable for $R_{\text {cache }}<$ $C_{W}\left(\mathbf{X}, D_{F}\right)$. On the contrary, for all $R_{\text {cache }} \geq C_{W}\left(\mathbf{X}, D_{F}\right)$ equality is achievable and this requires $d$ to be smaller than the condition mentioned in the theorem. Denote by $\mathbf{D}^{\prime}$ the diagonal matrix that solves $C_{W}\left(\mathbf{X}, D_{F}\right)$, then we have:

$$
\begin{aligned}
C_{W}\left(\mathbf{X}, D_{F}\right) & =\frac{1}{2} \log \frac{\left|\Sigma_{\mathbf{X}}\right|}{\left|\mathbf{D}^{\prime}\right|} \\
& \Downarrow \text { Hadamard (in)equality } \\
\prod_{i=1}^{K} D_{i, i}^{\prime} & =\left|\Sigma_{\mathbf{X}}\right| 2^{-2 C_{W}\left(\mathbf{X}, D_{F}\right)} .
\end{aligned}
$$

Hence, for $d^{\prime K}=\left|\Sigma_{\mathbf{X}}\right| 2^{-2 C_{W}\left(\mathbf{X}, D_{F}\right)}$ we have that $R_{\text {cache }}\left(d^{\prime}, D_{F}\right)=\frac{1}{2} \log \frac{\left|\Sigma_{\mathbf{X}}\right|}{d^{\prime K}}$, because it is the lower bound (63). For all $d$ smaller, the bound is also met with equality. Namely, there are infinitely many diagonal matrices $\mathbf{D}$ in the range of $D_{F} \cdot \mathbf{I} \preceq \mathbf{D} \preceq \mathbf{D}^{\prime}$ that are

1) achievable, because $\mathbf{D} \preceq \mathbf{D}^{\prime}$ and $\mathbf{D}^{\prime} \preceq \Sigma_{\mathbf{X}}$ implies that $\mathbf{D} \preceq \Sigma_{\mathbf{X}}$,

2) and can span all $\prod_{i=1}^{K} D_{i, i}^{\prime} \in\left[D_{F}^{K},\left|\Sigma_{\mathbf{X}}\right| 2^{-2 C_{W}\left(\mathbf{X}, D_{F}\right)}\right]$ and thus all $R_{\text {cache }} \in\left[C_{W}\left(\mathbf{X}, D_{F}\right), \frac{1}{2} \log \frac{\left|\Sigma_{\mathbf{X}}\right|}{D_{F}^{K}}\right]$.

If $D_{F} \leq \min \left(\operatorname{diag}\left(\mathbf{D}_{C_{W}}\right)\right)$, then $C_{W}(\mathbf{X})=C_{W}\left(\mathbf{X}, D_{F}\right)$ and $R_{\text {cache }} \geq C_{W}(\mathbf{X})$ is sufficient to achieve the lower bound (63).

Corollary 9. If $d \leq\left|\Sigma_{\mathbf{X}}\right| 2^{-\frac{2}{K} C_{W}\left(\mathbf{X}, D_{F}\right)}$, then the Gaussian caching rate-distortion function $R_{\text {cache }}\left(d, D_{F}\right)$ also characterizes the boundary of achievable $\left(R_{\text {cache }}, \bar{R}_{\text {update }}\right)$-pairs in general.

The use of Gaussian codebooks was an assumption in the desire to characterize the boundary of $\mathcal{R}_{\text {caching. Whenever }}$ $R_{\text {cache }} \geq C_{W}\left(\mathbf{X}, D_{F}\right)$ their use is, however, sufficient for 
optimality in general. The proof of the corollary is a direct extension of the two-dimensional case of Corollary 2 and is related to the separability of the Gaussian rate-distortion function over all links of the Gray-Wyner network. Figure 13 shows an example of Theorem 7 and Corollary 9 for a case where $D_{F} \leq \min \left(\operatorname{diag}\left(\mathbf{D}_{C_{W}}\right)\right)$.

\section{REFERENCES}

[1] M. A. Maddah-Ali and U. Niesen, "Fundamental limits of caching," IEEE Transactions on Information Theory, vol. 60, no. 5, pp. 28562867, May 2014.

[2] A. Hartung, "Netflix - the turnaround story of 2012!" Forbes, January 2013, [Online].

[3] G. J. Op 't Veld and M. C. Gastpar, "Caching (a pair of) gaussians," in 36th WIC Symposium on Information Theory in the Benelux, May 2015, pp. 4-11.

[4] - "Caching gaussians: Minimizing total correlation on the graywyner network," in 2016 Annual Conference on Information Science and Systems (CISS), March 2016, pp. 478-483.

[5] — - "Caching of bivariate gaussians with non-uniform preference probabilities," in 2017 Symposium on Information Theory and Signal Processing in the Benelux, May 2017, pp. 176-183.

[6] C. Y. Wang, S. H. Lim, and M. Gastpar, "Information-theoretic caching: Sequential coding for computing," IEEE Transactions on Information Theory, vol. 62, no. 11, pp. 6393-6406, Nov 2016.

[7] R. Timo, S. S. Bidokhti, M. A. Wigger, and B. C. Geiger, "A ratedistortion approach to caching," IEEE Transactions on Information Theory, vol. 64, no. 3, pp. 1957-1976, March 2018.

[8] R. Gray and A. Wyner, "Source coding for a simple network," Bell System Technical Journal, The, vol. 53, no. 9, pp. 1681-1721, Nov 1974.

[9] A. Wyner, "The common information of two dependent random variables," IEEE Transactions on Information Theory, vol. 21, no. 2, pp. 163-179, Mar 1975.

[10] S. Watanabe, "Information theoretical analysis of multivariate correlation," IBM Journal of Research and Development, vol. 4, no. 1, pp. 66-82, Jan 1960.

[11] K. Viswanatha, E. Akyol, and K. Rose, "The lossy common information of correlated sources," IEEE Transactions on Information Theory, vol. 60, no. 6, pp. 3238-3253, June 2014.

[12] G. Xu, W. Liu, and B. Chen, "A lossy source coding interpretation of wyner's common information," IEEE Transactions on Information Theory, vol. 62, no. 2, pp. 754-768, Feb 2016.

[13] U. Niesen and M. A. Maddah-Ali, "Coded caching with nonuniform demands," IEEE Transactions on Information Theory, vol. 63, no. 2, pp. 1146-1158, February 2017.

[14] M. Ji, A. M. Tulino, J. Llorca, and G. Caire, "Order-optimal rate of caching and coded multicasting with random demands," IEEE Transactions on Information Theory, vol. 63, no. 6, pp. 3923-3949, June 2017.

[15] P. Hassanzadeh, A. M. Tulino, J. Llorca, and E. Erkip, "On coding for cache-aided delivery of dynamic correlated content," IEEE Journal on Selected Areas in Communications, vol. 36, no. 8, pp. 1666-1681, August 2018.

[16] P. Hassanzadeh, A. M. Tulino, J. Llorca, and E. Erkip, "Rate-memory trade-off for caching and delivery of correlated sources," IEEE Transactions on Information Theory, vol. 66, no. 4, pp. 2219-2251, 2020.

[17] P. Hassanzadeh, E. Erkip, J. Llorca, and A. Tulino, "Distortion-memory tradeoffs in cache-aided wireless video delivery," in 2015 53rd Annual Allerton Conference on Communication, Control, and Computing (Allerton), Sept 2015, pp. 1150-1157.

[18] Q. Yang and D. Gündüz, "Centralized coded caching for heterogeneous lossy requests," in 2016 IEEE International Symposium on Information Theory (ISIT), July 2016, pp. 405-409.
[19] J. Xiao and Q. Luo, "Compression of correlated gaussian sources under individual distortion criteria," in 43rd Allerton Conference on Communication, Control, and Computing, 2005, pp. 438-447.

[20] W. Equitz and T. Cover, "Successive refinement of information," IEEE Transactions on Information Theory, vol. 37, no. 2, pp. 269-275, 1991.

[21] T. M. Cover and J. A. Thomas, Elements of information theory (2. ed.). Wiley, 2006.

[22] G. Xu, W. Liu, and B. Chen, "Wyners common information for continuous random variables - a lossy source coding interpretation," in 45th Annual Conference on Information Sciences and Systems (CISS), March 2011, pp. 1-6.

[23] J. Nayak, E. Tuncel, D. Gunduz, and E. Erkip, "Successive refinement of vector sources under individual distortion criteria," Information Theory, IEEE Transactions on, vol. 56, no. 4, pp. 1769-1781, April 2010.

[24] G. Xu, W. Liu, and B. Chen, "Wyner's common information: Generalizations and A new lossy source coding interpretation," CoRR, vol. abs/1301.2237, 2013. [Online]. Available: http://arxiv.org/abs/1301.2237

[25] W. Liu, G. Xu, and B. Chen, "The common information of $\mathrm{N}$ dependent random variables," in 2010 48th Annual Allerton Conference on Communication, Control, and Computing (Allerton), Sept 2010, pp. 836-843.

[26] L. Vandenberghe, S. Boyd, and S. P. Wu, "Determinant maximization with linear matrix inequality constraints," SIAM Journal on Matrix Analysis and Applications, vol. 19, no. 2, pp. 499-533, 1998. [Online]. Available: http://citeseerx.ist.psu.edu/viewdoc/summary?doi=10.1.1.46.1561

[27] S. Boyd and L. Vandenberghe, Convex Optimization. New York, NY, USA: Cambridge University Press, 2004.

Giel Op 't Veld received his M.S. degree in eletrical engineering from Eindhoven University of Technology (TU/e), Eindhoven, Netherlands, in 2012, and the Doctorat ès Science degree from the Ecole Polytechnique Fédérale (EPFL), Lausanne, Switzerland, in 2017. He since works as a management consultant at McKinsey \& Company.

Michael Gastpar received the Dipl. El.-Ing. degree from the Eidgenössische Technische Hochschule (ETH), Zürich, Switzerland, in 1997, the M.S. degree in electrical engineering from the University of Illinois at Urbana-Champaign, Urbana, IL, USA, in 1999, and the Doctorat ès Science degree from the Ecole Polytechnique Fédérale (EPFL), Lausanne, Switzerland, in 2002. He was also a student in engineering and philosophy at the Universities of Edinburgh and Lausanne.

During the years 2003-2011, he was an Assistant and tenured Associate Professor in the Department of Electrical Engineering and Computer Sciences at the University of California, Berkeley. Since 2011, he has been a Professor in the School of Computer and Communication Sciences, Ecole Polytechnique Fédérale (EPFL), Lausanne, Switzerland. He was also a professor at Delft University of Technology, The Netherlands, and a researcher with the Mathematics of Communications Department, Bell Labs, Lucent Technologies, Murray Hill, NJ. His research interests are in network information theory and related coding and signal processing techniques, with applications to sensor networks and neuroscience.

Dr. Gastpar received the IEEE Communications Society and Information Theory Society Joint Paper Award in 2013 and the EPFL Best Thesis Award in 2002. He was an Information Theory Society Distinguished Lecturer (2009-2011), an Associate Editor for Shannon Theory for the IEEE TRANSACTIONS ON INFORMATION THEORY (2008-2011), and he has served as Technical Program Committee Co-Chair for the 2010 International Symposium on Information Theory, Austin, TX. 